\title{
Two-Phase Free Boundary Problems for Parabolic Operators: Smoothness of the Front
}

\author{
FAUSTO FERRARI \\ Università di Bologna \\ SANDRO SALSA \\ Politecnico di Milano
}

\begin{abstract}
We continue to develop the regularity theory of general two-phase free boundary problems for parabolic operators. In a 2010 paper, we establish the optimal (Lipschitz) regularity of a viscosity solution under the assumptions that the free boundary is locally a flat Lipschitz graph and a nondegeneracy condition holds.

Here, on one side we improve this result by removing the nondegeneracy as-sumption; on the other side we prove the smoothness of the front. The proof relies in a crucial way on a local stability result stating that, for a certain class of operators, under small perturbations of the coefficients flat free boundaries remain close and flat.
\end{abstract}

\section{Introduction and Main Results}

In this paper we continue to develop the regularity theory of general two-phase free boundary problems for parabolic operators started in [11]. Specifically, let $\mathscr{C}_{R, T}=B_{R}^{\prime} \times(-T, T)$, where $B_{R}^{\prime}$ is the ball in $\mathbb{R}^{n}$ of radius $R$ centered at the origin, and

$$
\mathscr{L}=\operatorname{Tr}\left(A(x, t) D^{2}\right)+\mathbf{b}(x, t) \cdot \nabla=\sum_{i, j=1}^{n} a_{i j}(x, t) D_{i j}+\sum_{j=1}^{n} b_{j}(x, t) D_{j} .
$$

We assume that $a_{i j} \in C^{0, \alpha}\left(\overline{\mathscr{C}}_{R, T}\right), 0<\alpha \leq 1$, for every $1 \leq i, j \leq n$, with respect to the parabolic distance, and that there exist $\lambda, \Lambda>0$ such that for every $\xi \in \mathbb{R}^{n}$ and for every $(x, t) \in \mathscr{C}_{R, T}$

$$
\lambda|\xi|^{2} \leq \sum_{i, j=1}^{n} a_{i j}(x, t) \xi_{i} \xi_{j} \leq \Lambda|\xi|^{2} .
$$

Moreover, we can assume that $A(0,0)=I$. For the modulus of continuity of the coefficients $a_{i j}$, we u se the symbol $\omega(r)=\sup _{\delta_{p} \leq r}|A(p)-I|$, where $\delta_{p}$ denotes the parabolic distance of $p=(x, t) \in \mathbb{R}^{n+1}$ from $(0,0)$. We also assume 
for simplicity $b_{j} \in C^{0, \alpha}\left(\overline{\mathscr{C}}_{R, T}\right)$; see, however, Remark 1.3 . Let $\mathscr{A}$ be the set of operators satisfying the above hypotheses.

The class of free boundary problems (f.b.p.) we shall deal with can be formally stated in the following way: to find a function $u \in C\left(\mathscr{C}_{R, T}\right)$ such that

and

(i) $\begin{cases}\mathscr{L} u-D_{t} u=0 & \text { in } \Omega^{+}(u)=\mathscr{C}_{R, T} \cap\{u>0\}, \\ \mathscr{L} u-D_{t} u=0 & \text { in } \Omega^{-}(u)=\mathscr{C}_{R, T} \cap\{u \leq 0\}^{o},\end{cases}$

(ii) $V_{v}=-G\left(\left|\nabla u^{+}\right|,\left|\nabla u^{-}\right|\right)$

on $F(u)=\partial \Omega^{+}(u) \cap \mathscr{C}_{R, T}$, the so-called free boundary condition, where $V_{v}=$ $V_{\nu}(\cdot, \tau)$ is the speed of the surface $F_{\tau}(u)=F(u) \cap\{t=\tau\}$ in the direction $v=\nabla u^{+} /\left|\nabla u^{+}\right|$.

The basic requirements on the function $G$ express that $V_{v}$ is controlled in an "elliptic" way, i.e., monotonically in the flux discontinuity:

(a) $G=G(a, b)$ is a Lipschitz function in $[0,+\infty) \times[0,+\infty)$.

(b) There exists a constant $c_{G}$ such that

$$
D_{a} G,-D_{b} G \geq c_{G}>0,
$$

uniformly in $\in[0,+\infty) \times[0,+\infty)$;

(c) $G \rightarrow+\infty$ when $a-b \rightarrow+\infty$.

By a classical subsolution (supersolution, solution) of the above problem, we mean a function $u \in C\left(\mathscr{C}_{R, T}\right)$ such that

(i-cl) $u \in C^{1}\left(\bar{\Omega}^{+}(u)\right) \cap C^{2}\left(\Omega^{+}(u)\right)$ and $u \in C^{1}(\bar{\Omega}(u)) \cap C^{2}\left(\Omega^{-}(u)\right)$,

$$
\begin{array}{ll}
\mathscr{L} u-D_{t} u \geq 0(\leq 0,=0) & \text { in } \Omega^{+}(u)=\mathscr{C}_{R, T} \cap\{u>0\}, \\
\mathscr{L} u-D_{t} u \geq 0(\leq 0,=0) & \text { in } \Omega^{-}(u)=\mathscr{C}_{R, T} \cap\{u \leq 0\}^{\circ},
\end{array}
$$

(ii-cl) the free boundary $F(u)$ is a $C^{1}$ surface, $\left|\nabla u^{+}\right|>0$ on $F(u)$, and for every $(x, t) \in F(u)$,

$$
-V_{v}=\frac{D_{t} u^{+}}{\left|\nabla u^{+}\right|} \leq G\left(\left|\nabla u^{+}\right|,\left|\nabla u^{-}\right|\right) \quad(\geq,=) .
$$

Viscosity solutions are defined in the following way (see [6]):

DeFinition 1.1. Let $u$ be a continuous function in $\mathscr{C}_{R, T}$. We say that $u$ is a viscosity subsolution (supersolution) of our f.b.p. if, for any subcylinder $\mathscr{C}$ of $\mathscr{C}_{R, T}$ and for every classical $C^{2}$-supersolution (subsolution) $v$ in $\mathscr{C}, u \leq v(u \geq v)$ on the parabolic boundary $\partial_{p} \mathscr{C}$ implies that then $u \leq v(u \geq v)$ in $\mathscr{C}$. The function $u$ is called a viscosity solution if it is both a viscosity supersolution and a viscosity subsolution.

Existence results for viscosity solutions can be found in [14]; actually, in that very nice paper, the authors give a slightly different notion of viscosity solution and also prove a comparison theorem for subsolutions and supersolutions with 
strictly separated boundary data. All the above results are still valid in our case and, in particular, we will use their comparison theorem 3.1, in Section 4.1. In the case $\mathscr{L}=\Delta$ and $G(a, b)=a-b$, that is, in the classical two-phase Stefan problem, they also prove the equivalence of the notions of viscosity solution and weak solution defined via the so-called enthalpy formulation (see, e.g., [12,17]). A remarkable consequence is the uniqueness of viscosity solutions with continuous boundary data.

Some related results concerning questions of uniqueness in two-phase problems are shown in [18]. In particular, via a very interesting comparison theorem, uniqueness of a viscosity solution is established in singular perturbation problems for a class of fully nonlinear operators of the form $F\left(D w, D^{2} w\right)$, including, for instance, the $p$-Laplace operator.

As far as the regularity of the solution and the free boundary are concerned, another recent important paper is [19]. In that paper the authors prove that the classical two-phase Stefan problem admits a unique local (in time) solution that is analytic in space and time, under mild assumptions on the regularity and nondegeneracy of the initial data.

For viscosity solutions, the papers $[3-5]$ deal with the optimal regularity of a solution and the analysis of the free boundary for nonlinear general $G$ and $\mathscr{L}=\Delta$. These results are of a local nature and are therefore independent of boundary or initial data. In particular, in [3] it is shown that if the free boundary is locally a Lipschitz graph in space and time, then the solution is Lipschitz across the free boundary. Note that the Lipschitz continuity in time corresponds to finite speed of the free boundary.

On the other hand, Lipschitz continuity alone cannot give any further regularization of the free boundary as the counterexample in [4] shows. Actually, an analogous counterexample holds even for a one-phase Stefan problem (see [2,20]).

To achieve further regularization of the free boundary it is enough to add a nondegeneracy condition preventing the two fluxes from vanishing simultaneously on both sides of the free boundary. It is remarkable that the same effect can be obtained by asking that the time sections of the free boundary be flat, that is, that the Lipschitz constant in space be sufficiently small or that the solution be $\epsilon$-monotone (see the definition below) along the directions of a space-time cone whose time section is sufficiently open. This situation occurs, for instance, in the limiting behavior of blowup sequences of the type $\rho^{-1} u(\rho x, \rho t)$ as $\rho \rightarrow 0$. This fact will play a crucial role in Section 4.

Under the above conditions, the free boundary is locally a $C^{1}$ graph, the nondegeneracy condition $\left|\nabla u^{+}\right|>0$ holds on $F(u)$, and therefore a viscosity solution is indeed a $C^{1}$-classical solution. In [15] it is shown that if $G$ is smooth, nondegenerate $C^{1}$-free boundaries are indeed $C^{\infty}$.

When $\mathscr{L}$ has the general form (1.1) the only known result is in [11], where we prove the Lipschitz continuity of the solution under the hypotheses that the free boundary is flat in space and that a nondegeneracy condition holds. Here we get 
rid of this nondegeneracy condition and prove directly that the free boundary is $C^{1}$ and that viscosity solutions are indeed classical solutions.

We can now state our main result for a true two-phase problem, which is the significant case. The extension to the one-phase case is straightforward. We denote by $p=(x, t)=\left(x^{\prime}, x_{n}, t\right)$ points in $\mathbb{R}^{n-1} \times \mathbb{R} \times \mathbb{R}$.

THEOREM 1.2. Let $u$ be a viscosity solution to our f.b.p. in $\mathscr{C}_{2,2}$ and set $M=$ $\sup _{\mathscr{C}_{2,2}}|u|,\left|u\left( \pm e_{n},-3 / 2\right)\right| \geq m>0$. Let the free boundary $F(u)$ be given by the graph of a function $x_{n}=f\left(x^{\prime}, t\right)$ with $(0,0) \in F(u)$. Assume that there exist $L$, $L_{0} \geq 0$, such that for every $(x, t),(y, s) \in \mathscr{C}_{2,2} \cap F(u)$,

$$
\left|f\left(x^{\prime}, t\right)-f\left(y^{\prime}, s\right)\right| \leq L\left|x^{\prime}-y^{\prime}\right|+L_{0}|t-s| .
$$

If $L$ is small enough, depending only on $n,\|A\|_{C^{0, \alpha}},\|\mathbf{b}\|_{\infty}, L_{0}, \lambda, \Lambda, m / M, c_{G}$, then $\mathscr{C}_{1,1} \cap F(u)$ is a $C^{1}$-graph in space and time. Moreover, there exist positive constants $c_{1}, c_{2}$ depending only on $n,\|A\|_{C^{0, \alpha}},\|\mathbf{b}\|_{\infty}, L_{0}, \lambda, \Lambda, m / M$, and $c_{G}$ such that, for every $\left(x^{\prime}, x_{n}, t\right),\left(y^{\prime}, y_{n}, s\right) \in F(u)$ :

(i) $\left|\nabla_{x^{\prime}} f\left(x^{\prime}, t\right)-\nabla_{x^{\prime}} f\left(y^{\prime}, s\right)\right| \leq c_{1}\left(\log \left|x^{\prime}-y^{\prime}\right|\right)^{-4 / 3}$,

(ii) $\left|D_{t} f\left(x^{\prime}, t\right)-D_{t} f\left(x^{\prime}, s\right)\right| \leq c_{1}\left(\log \left|t-s^{\prime}\right|\right)^{-1 / 3}$.

As a consequence, $u \in C^{1}\left(\bar{\Omega}^{+}(u)\right) \cap C^{1}(\bar{\Omega}(u))$ on $F(u)$ and

$$
\left|\nabla u^{+}\right| \geq c_{2}>0 \text {. }
$$

As a byproduct of the proof of Theorem 1.2 we obtain an interior stability result for the free boundary that could be of independent interest (see Theorem 4.2).

The overall strategy of the proof of Theorem 1.2 follows the papers [4,5] and is based on perturbation from the constant-coefficient case with two main elements of novelty that we are going to describe along with the general lines of the proofs commenting on the relevance and the role of the various hypotheses.

First, we make a few remarks on rescalings. As is common in regularity theories, the above results are obtained through an iteration process involving a sequence of rescalings of the problem. In principle, there are three types of rescaling that we could use: a parabolic rescaling, $u_{\rho}(x, t)=u\left(\rho x, \rho^{2} t\right)$; a parabolic blowup, $u_{\rho}(x, t)=\rho^{-1} u\left(\rho x, \rho^{2} t\right)$; and a hyperbolic blowup, $u_{\rho}(x, t)=\rho^{-1} u(\rho x, \rho t)$. Here we assume that $\rho \rightarrow 0$.

The parabolic rescaling leaves the equation and, if $G$ is linear, the free boundary condition unchanged. However, it progressively deteriorates the underlying nondegeneracy condition hidden inside the flatness condition, namely a Hopf-type principle at regular points of the free boundary (see Lemma 6.1 below or lemma 5.1 in [5]). A point $\left(x_{0}, t_{0}\right) \in F(u)$ is regular from the right (respectively, left) if there exists an $(n+1)$-dimensional ball $B \subset \Omega^{+}(u)$ (respectively, $\left.\Omega^{-}(u)\right)$ such that $\left\{\left(x_{0}, t_{0}\right)\right\}=\partial B \cap F(u)$.

We use this rescaling only once at the beginning of the proof, to get the $\epsilon_{0}$ monotonicity of $u$ along a space-time cone $\Gamma^{t}\left(e_{n}, \theta^{t}\right)$, where $\epsilon_{0}$ is a small positive number and $\theta^{t} \geq \frac{\pi}{4}$. 
After a parabolic blowup, the equation and the nondegeneracy condition remain unaltered, but the free boundary condition progressively degenerates, preventing any gain in regularity (see the counterexample in [4]) and any error control in the perturbation arguments.

The hyperbolic blowup leaves nondegeneracy and the free boundary condition unchanged. Also the perturbation error can easily be kept under control. However, there are two drawbacks. A minor one is that the coefficient of $D_{t} u_{\rho}$ is vanishing, disconnecting more and more the various time levels. This inconvenience is kept under control during the iterations by the space-time monotonicity properties of $u$. A more serious problem is that every estimate has to be done in hyperbolic geometry. As in [4, 5] this forces us to use a intermediate, almost-hyperbolic blowup tailored to overcome the above difficulty.

The plan of the paper is the following.

In Section 2] we recall some results from [11] about the full monotonicity of $u$ along the direction of a spacial cone $\Gamma^{x}\left(e_{n}, \theta\right)$ of axis $e_{n}$ and opening $\theta$, its $\epsilon_{0^{-}}$ monotonicity and its full monotonicity $\epsilon_{0}$-away from the free boundary, along the direction of a space-time cone $\Gamma^{t}\left(e_{n}, \theta^{t}\right)$, with $\mu=\frac{\pi}{2}-\theta^{t} \ll \delta=\frac{\pi}{2}-\theta$. Notice that this gives a control of $D_{t} u$ in terms of $D_{n} u, \epsilon_{0}$-away from the free boundary.

Clearly, the defect angles $\delta$ and $\mu$ measure the flatness of the level surfaces of $u$. The next goal is to decrease these defects away from the free boundary. This must be done in the above almost-hyperbolic homogeneity and, as in the constantcoefficient case, the free boundary condition has to play a role. Since directional derivatives do not satisfy any kind of reasonable equation, one has to resort to a quite delicate perturbation argument based on two key facts.

The first one is that the parabolic version of the sup-inf convolution family of deformations constructed in [10] gives subsolutions for a single operator and also for a class of slightly perturbed operators belonging to $\mathscr{A}$ (Section 3 ).

The second one is a local stability result: the free boundary of a solution $z$ of the f.b.p. for the heat operator sharing with $u$ the boundary data is contained in an $\epsilon_{0}$-neighborhood of $F(u)$ (see Theorem 4.1). Thus, thanks to [5], the function $z$ becomes a classical solution. Here the Hopf principle at right and left (unless $u^{-} \equiv 0$ ) regular points plays a crucial role. We can then use $z$ as a perturbation of $u$ to get the proper gain in monotonicity through the corresponding properties of $z$ itself (Section 4).

At this point one has all the ingredients to apply the results in [4] in order to propagate the gains in the opening of the space and space-time cones of $\epsilon_{0^{-}}$ monotonicity to the free boundary in a smaller cylinder with the proper homogeneity.

Still we have $\epsilon_{0}$-monotonicity only, so that we need to decrease $\epsilon_{0}$. This can be done exactly as in [11] (Section 3) through the construction of another family of deformations; this is possible thanks to the progressive improvement of the cones opening. 
In both the gains of the cones opening and of $\epsilon_{0}$-monotonicity, the Hopf principle is once more crucial (although this time only for right-regular points).

Thus, the initial step in the iteration is proved. At this point, a double iteration procedure as in [5] can be performed to achieve the results in Theorem 1.2. at each step we obtain a cone enlargement (from $\delta_{k}$ to $\delta_{k+1}<\delta_{k}$, from $\mu_{k}$ to $\mu_{k+1}<\mu_{k}$ ) and an $\epsilon_{0}$-monotonicity improvement (from $\epsilon_{k}$ to $\epsilon_{k+1}<\epsilon_{k}$ ) in a sequence of contracting cylinders, whose homogeneity turns out to be intermediate between parabolic and hyperbolic. This produces a speed of the cones opening corresponding to a logarithmic modulus of continuity for $\nabla f, D_{t} f$. Particular care is required in keeping under control the balance between $\epsilon_{k}$ and the smallness of $\|A-I\|_{\infty}$ and $\|\mathbf{b}\|_{\infty}$ at each step.

Remark 1.3. The above result holds for operators with a drift term $\mathbf{b}(x, t) \cdot \nabla u$ as well when $\mathbf{b}$ is merely bounded measurable. Indeed, we work with $b_{j} \in C\left(\overline{\mathscr{C}}_{R, T}\right)$ only to avoid the technicalities of $L^{p}$-viscosity solutions. Thus, our results hold in particular for operators in divergence form with Lipschitz coefficients, where $\left|\nabla u^{ \pm}\right|$are replaced by the conormal derivatives in the free boundary condition. To handle this case, the only difference lies in the construction of the families of appropriate sup-inf convolutions, where we have to use a viscosity approach as in [10]. Semilinear operators of the form

$$
\mathscr{L}=\operatorname{Tr}\left(A(u, x, t) D^{2}\right)+\mathbf{b}(u, x, t) \cdot \nabla
$$

where $A$ is Hölder-continuous with respect to all its arguments and $\mathbf{b}$ is bounded are also included.

Remark 1.4. The extension to a more general free boundary condition

$$
V_{v}=-G\left(\left|\nabla u^{+}\right|,\left|\nabla u^{-}\right|, v, x\right)
$$

is possible if $G$ is Lipschitz with respect to $v, x$, with small Lipschitz constant depending on $L, L_{0}$. This smallness condition actually enters only in the first step of the iteration process.

The various constants $c, C$ that will appear in the following may vary from formula to formula. If for a constant $c, \eta, \kappa$, etc., we don't give any explicit dependence, we mean that it depends only on some of the relevant parameters $n$, $\|A\|_{C^{0, \alpha}},\|\mathbf{b}\|_{\infty}, L, L_{0}, \lambda, \Lambda, m / M$, and $c_{G}$.

\section{$2 \mathscr{L}$-Caloric Functions in Lipschitz Domains: Known Results}

In this section we collect for the reader's convenience some well-known Harnack principles and some of the results in [11] more or less continuously quoted in this paper.

Following [6], by a $\mathscr{L}$-caloric function we mean a nonnegative solution $u$ of the equation $\mathscr{L} u-D_{t} u=0$ in some Lipschitz domain $\Psi \subset \mathbb{R}^{n+1}$ that vanishes locally on some distinguished part of $\partial \Psi$. Specifically, let

$$
\Psi_{2 r}=\left\{\left(x^{\prime}, x_{n}, t\right):\left|x^{\prime}\right|<2 L^{-1} r,|t|<4 L_{0}^{-2} r^{2}, f\left(x^{\prime}, t\right)<x_{n}<4 r\right\}
$$


and $u$ be $\mathscr{L}$-caloric in $\Psi_{2}$ vanishing on

$$
F(u)=\left\{\left(x^{\prime}, x_{n}, t\right):\left|x^{\prime}\right|<2 L^{-1},|t|<4 L_{0}^{-2}, x_{n}=f\left(x^{\prime}, t\right)\right\} .
$$

We always assume that $f(0,0)=0$. Let $P_{r}=(0, r, 0), \bar{P}_{r}=\left(0, r, 2 L_{0}^{-2} r^{2}\right)$, and $\underline{P}_{r}=\left(0, r,-2 L_{0}^{-2} r^{2}\right)$. Moreover, let $m=u\left(\underline{P}_{3 / 2}\right)>0$ and $M=\sup _{\Psi_{2}} u$ finite.

\section{Harnack Principles}

We will repeatedly use the interior Harnack inequality and some boundary Harnack principles. We recall these results in a form adapted to our situation.

Let $\delta((x, t),(y, s))=|x-y|+|t-s|^{1 / 2}$ be the parabolic distance and set

$$
\delta_{x, t}=\delta((x, t),(0,0)) \text {. }
$$

Then we have:

INTERIOR HARNACK INEQUALITY (SEE [16]): There exists a positive constant $c=c(n, \lambda, \Lambda)$ such that for any $r \in(0,1)$ :

$$
u\left(\underline{P}_{r}\right) \leq c u\left(\bar{P}_{r}\right) .
$$

Carleson Estimate (SeE [13]): There exist $c=c\left(n, \lambda, \Lambda, L, L_{0}\right)>0$ and $\beta=\beta\left(n, \lambda, \Lambda, L, L_{0}\right), 0<\beta \leq 1$, such that for every $(x, t) \in \Psi_{r / 2}$

$$
u(x, t) \leq c\left(\frac{\delta_{x, t}}{r}\right)^{\beta} u\left(\bar{P}_{r}\right) .
$$

Boundary Harnack PRinciple (SEe [8]): There exist $c=c(n, \lambda, \Lambda, L$, $\left.L_{0}\right)>0$ and $\beta=\beta\left(n, \lambda, \Lambda, L, L_{0}\right), 0<\beta \leq 1$, such that for every $(x, t) \in \Psi_{r / 2}$

$$
\frac{u(x, t)}{v(x, t)} \geq c \frac{u\left(\underline{P}_{r}\right)}{v\left(\bar{P}_{r}\right)} .
$$

BACKWARD HARNACK INEQUALITY (SEE [3]): There exists a positive constant $c=c\left(n, \lambda, \Lambda, L, L_{0}, m / M\right)$ such that if $r<\frac{1}{2}$

$$
u\left(\bar{P}_{r}\right) \leq c u\left(\underline{P}_{r}\right) .
$$

The proof of [2.3) follows the same line in [3], using the results in [1, 8, 9] on the $\mathscr{L}$-caloric measure .

\section{Monotonicity in Space}

If $u$ is $\mathscr{L}$-caloric, then it is monotone increasing along a spatial cone of directions. We shall denote by $\Gamma^{x}(e, \theta)$ a spatial cone with opening $\theta$ and axis $e$; in particular, if $\tau \in \Gamma^{x}(e, \theta)$, then $\left\langle\tau, e_{t}\right\rangle=0$ where $e_{t}$ is the time direction. Moreover, $\Gamma^{t}(v, \theta)$ will denote a space-time cone of width $\theta$ and axis $v \in \operatorname{span}\left\{e, e_{t}\right\}$. In such case a vector $\tau \in \Gamma^{t}(v, \theta)$ can have both space and time components. Finally, set

$$
F_{s}(u)=F(u) \cap\{t=s\} .
$$

Then we have the following (see lemma 2.1 in [11]): 
Lemma 2.1. There exists $\eta=\eta\left(n, L, L_{0},\|A\|_{C^{0, \alpha}},\|\mathbf{b}\|_{\infty}, m / M, \lambda, \Lambda\right)>0$ such that in $\Psi_{1} \cap\left\{d_{x, t} \leq \eta\right\}, u$ is increasing along the space directions $\tau$ belonging to the cone $\Gamma^{x}\left(e_{n}, \theta\right)$, with opening $\theta=\frac{1}{2} \cot ^{-1}$ L. Moreover, there exist positive constants $c_{1}, c_{2}$, depending only on $n, L, L_{0},\|A\|_{C^{0, \alpha}},\|\mathbf{b}\|_{\infty}, m / M, \lambda$, and $\Lambda$ such that, in $\Psi_{1} \cap\left\{d_{x, t} \leq \eta\right\}$,

$$
c_{1} \frac{u(x, t)}{d_{x, t}} \leq D_{n} u(x, t) \leq c_{2} \frac{u(x, t)}{d_{x, t}},
$$

where $d_{x, t}=\operatorname{dist}\left((x, t), F_{t}(u)\right)$.

\section{Space-Time $\epsilon$-Monotonicity and Full Monotonicity}

We say that $u \geq 0$ is $\epsilon$-monotone along a direction $\tau \in \mathbb{R}^{n+1}$ in $\Psi_{1}$ if

$$
u\left((x, t)+\epsilon^{\prime} \tau\right)-u(x, t) \geq 0
$$

for every $\epsilon^{\prime} \geq \epsilon$ as long as $(x, t)$ and $(x, t)+\epsilon^{\prime} \tau$ both belong to $\Psi_{1}$.

We can express the $\epsilon$-monotonicity along all the directions $\tau \in \Gamma\left(e^{*}, \theta^{*}\right)$ by saying, in an essentially equivalent way, that for every $\epsilon^{\prime} \geq \epsilon$ and every $\tau \in$ $\Gamma\left(e^{*}, \theta^{*} / 2\right)$

$$
u_{\epsilon^{\prime}}(p) \equiv \sup _{q \in B_{\epsilon^{\prime} \sin \theta^{*} / 2}(p)} u\left(q-\epsilon^{\prime} \tau\right) \leq u(p)
$$

for all $p=(x, t)$ at distance greater than $\epsilon^{\prime}$ from $\partial \Psi_{1}$.

Let $\epsilon_{0}$ be a small number to be chosen later depending only on $n, L, L_{0}$, $\|A\|_{C^{0, \alpha}},\|\mathbf{b}\|_{\infty}, m / M, \lambda$, and $\Lambda$. After a suitable parabolic rescaling, we can rewrite lemma 2.3 in [11] in the following way:

LeMma 2.2. Let $\eta$ be as in Lemma 2.1 and $\epsilon_{0}<\eta / 100$. There exist positive constants $C_{0}, \theta^{t}$, and $\frac{\pi}{4} \leq \theta^{t}<\frac{\pi}{2}$, depending only on $n, L, L_{0},\|A\|_{C^{0, \alpha}},\|\mathbf{b}\|_{\infty}$, $m / M, \lambda$, and $\Lambda$ such that

(a) in $\Psi_{1} \cap\left\{d_{x, t} \leq \eta\right\}$

$$
\left|D_{t} u(x, t)\right| \leq C_{0} d_{x, t}^{a-1} D_{n} u(x, t),
$$

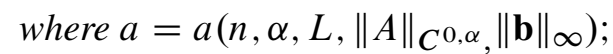

(b) $u$ is $\epsilon_{0}$-monotone in $\Psi_{1} \cap\left\{d_{x, t} \leq \eta\right\}$ and fully monotone in $\Psi_{1} \cap\left\{\epsilon_{0} \leq\right.$ $\left.d_{x, t} \leq \eta\right\}$ along the directions of a space-time cone $\Gamma^{t}\left(e_{n}, \theta^{t}\right)$.

Remark 2.3. After the above-mentioned rescaling, we can actually assume $\eta=1$ in Lemmas 2.1 and 2.2. and moreover $\omega(r) \leq c r_{0}^{\alpha} r^{\alpha}$ with $r_{0} \leq \epsilon_{0}^{(1-\alpha) / \alpha}$. Also, we can assume $\|\mathbf{b}\|_{\infty} \leq \omega(1)$. This will be used later on.

\section{Families of Continuous Deformations}

In this section we construct two families of continuous deformations that play a key role in our regularization procedure. The main point is that these families turn out to be families of sub/supersolutions for a single operator and also for a set of operators with close coefficients. We need this to get the improvement of 
the smoothness of the level sets far from the free boundary in properly scaling regions. In the variable-coefficient case this step constitutes a major difficulty, since derivatives do not satisfy any reasonable parabolic equation.

In the following we shall denote by $\mathscr{P}^{-}$the extremal Pucci's operator defined as

$$
\mathscr{P}^{-}(\phi)=\inf _{A \in \mathscr{S}_{\lambda, \Lambda}} \operatorname{Tr}\left(A D^{2} \phi\right),
$$

where $\mathscr{S}_{\lambda, \Lambda}$ is the class of symmetric matrices with eigenvalues between $\lambda$ and $\Lambda$, $0<\lambda \leq \Lambda$.

\subsection{Subsolutions and Supersolutions for Parabolic Operators}

For a diffusion matrix $A$ and drift $\mathbf{b}$ we denote by $\mathscr{L}_{A, \mathbf{b}}$ the corresponding parabolic operator in $\mathscr{A}$. Let $u$ be a solution of our f.b.p. in a cylinder $\mathscr{C}_{R, T}$, and $\phi$ be a smooth function such that

$$
v_{\phi}(p)=\sup _{B_{\phi(p)}(p)} u=\sup _{|\xi|=1} u(p+\phi(p) \xi)
$$

is well-defined in some region $D \subset \overline{\mathscr{C}}_{R, T} \subset \mathbb{R}^{n+1}$. Assume that $P=p+$ $\phi(p) v^{*}$, where $v^{*} \in \mathbb{R}^{n+1}$ and $\left|v^{*}\right|=1$, is the point where the maximum is achieved so that $v_{\phi}(p)=u(P)$. Observe that if $v^{*}=v_{s}^{*}+v_{t}^{*}$, where $v_{s}^{*}=$ $\left(v_{1}^{*}, \ldots, v_{n}^{*}, 0\right)$ and $v_{t}^{*}=\left(0, \ldots, 0, v_{n+1}^{*}\right)$, then $\left|v_{s}^{*}\right|=\sqrt{1-\left(v_{t}^{*}\right)^{2}}$ and

$$
\begin{aligned}
v_{\phi}(p) & =\sup \left\{u(q): q \in B_{\left|v_{s}^{*}\right| \phi(p)}^{\prime}\left(p+v_{t}^{*} \phi(p)\right)\right\} \\
& =\sup _{\sigma \in B_{\left|\nu_{s}^{*}\right| \phi(p)}^{\prime}}^{\prime} u(p)
\end{aligned}
$$

For a small vector $\tau$ define

$$
v_{\phi, \tau}(p)=\sup _{B_{\phi(p)}(p)} u(q-\tau)=\sup _{|\xi|=1} u(p-\tau+\phi(p) \xi)=u\left(P_{\tau}\right),
$$

where $P_{\tau}=p-\tau+\phi(p) v$ for some suitable vector $v$. Similarly, we set

$$
w_{\phi, \tau}(p)=\inf _{B_{\phi(p)}(p)} u(q-\tau)=\inf _{|\xi|=1} u(p-\tau+\phi(p) \xi)=u\left(\widetilde{P}_{\tau}\right),
$$

where $\widetilde{P}_{\tau}=p-\tau+\phi(p) \widetilde{v}^{*}$ for some $\widetilde{v}^{*},\left|\widetilde{v}^{*}\right|=1$.

THEOREM 3.1. Let $u$ be a solution of our f.b.p. in a cylinder $\mathscr{C}_{R, T}$ for the operator $\mathscr{L}_{B, \mathbf{b}}-D_{t}$. Assume that $\mathscr{L}_{A, \mathbf{b}^{\prime}}-D_{t}$ is another operator in the same class. Let $\epsilon_{0}$ be as in Lemma 2.2 and $\phi \in C^{2}\left(\overline{\mathscr{C}}_{R, T}\right)$ be a strictly positive function. Let $\omega \leq \omega\left(\phi_{\max }\right)$, where $\phi_{\max }=\max _{\overline{\mathscr{C}}_{R, T}} \phi$. Assume that

$$
\|A-B\|_{\infty} \leq C_{0}\left(\frac{\Lambda}{\lambda}-1\right) \leq \omega, \quad\left\|\mathbf{b}-\mathbf{b}^{\prime}\right\|_{\infty} \leq \omega,
$$

and that in a smaller cylinder $\mathscr{C}^{\prime} \subset \mathscr{C}_{R, T}$, with $\operatorname{dist}\left(\mathscr{C}^{\prime}, \mathscr{C}_{R, T}\right) \geq \rho \gg \epsilon_{0}$,

$$
D_{t} \phi \geq 0
$$


and

$$
\mathscr{P}^{-}(\phi)-c_{1} D_{t} \phi \geq C \frac{|\nabla \phi|^{2}+\omega^{2}}{\phi}+c_{2}(|\nabla \phi|+\omega)
$$

for some positive constants $C_{0}, C, c_{1}$, and $c_{2}$ depending only on $n, \lambda, \Lambda$, and $\rho$. Then

(i) in both $\Omega^{ \pm}\left(v_{\phi, \tau}\right) \cap \mathscr{C}^{\prime}, v_{\phi, \tau}$ is a viscosity subsolution for the operator $\mathscr{L}_{A, \mathbf{b}^{\prime}}-D_{t}$

(ii) in both $\Omega^{ \pm}\left(w_{\phi, \tau}\right) \cap \mathscr{C}^{\prime}, w_{\phi, \tau}$ is a viscosity supersolution for the operator $\mathscr{L}_{A, \mathbf{b}^{\prime}}-D_{t}$.

Remark 3.2. After Remark 2.3, if $\phi$ satisfies (3.2), then also $\epsilon_{0} \phi$ satisfies the same inequality, with a slightly different constant independent of $\epsilon_{0}$.

Since the proof of Theorem 3.1 is very technical, we have postponed it to the Appendix.

\subsection{Construction of the Variable Radius}

We now proceed to construct a family of radii $\phi_{\eta}$ with $D_{t} \phi_{\eta} \geq 0$ and satisfying in particular 3.2.

Lemma 3.3. Let $0<T$ and $C>1$. There exist positive constants $\bar{C}=\bar{C}(T, C)$, $k=(T, C)$, and $h_{0}=h_{0}(T, C)$ such that for any $0<h<h_{0}$, there is a family of $C^{2}$-continuous functions $\phi_{\eta}, 0 \leq \eta \leq 1$, defined in the closure of

$$
D=\left[B_{1}^{\prime} \backslash\left(\bar{B}_{1 / 8}^{\prime}\left(\frac{3}{4} e_{n}\right) \cup \bar{B}_{1 / 8}^{\prime}\left(-\frac{3}{4} e_{n}\right)\right)\right] \times(-T, T)
$$

such that

(i) $1-\omega \leq \phi_{\eta} \leq 1+\eta h$ in $\bar{D}$,

(ii) $\mathscr{P}^{-}\left(\phi_{\eta}\right)-c_{1} D_{t} \phi_{\eta}-C \frac{\left|\nabla \phi_{\eta}\right|^{2}+\omega^{2}}{\phi_{\eta}}-c_{2}\left(\left|\nabla \phi_{\eta}\right|+\omega\right) \geq 0$ in $D$,

(iii) $\phi_{\eta} \geq 1+k \eta h$ in $B_{1 / 2}^{\prime} \times\left(-\frac{T}{2}, \frac{T}{2}\right)$,

(iv) $\phi_{\eta} \leq 1$ in $D \backslash\left(B_{7 / 8}^{\prime} \times\left(-\frac{7 T}{8}, \frac{7 T}{8}\right)\right)$,

(v) $D_{t} \phi_{\eta} \leq \bar{C} \eta h$ and $\left|\nabla \phi_{\eta}\right| \leq \bar{C}(\eta h+\omega)$ in $\bar{D}$, and

(vi) $D_{t} \phi_{\eta} \geq 0$ in $D$,

for $c_{1}, c_{2}$, and $\omega$ small positive constants depending on $n, \lambda, \Lambda$, and $C$.

PROOF. We split the proof into two parts. In the first part we construct a function $\varphi_{\eta}$ that satisfies our theorem when $\omega=0$, while in the second one we modify $\varphi_{\eta}$ in order to satisfy our theorem.

Step 1 . We construct $\varphi_{\eta}=\left(1+\psi_{\eta}\right)^{1 /(1-C)}$, where $C>1$ has to be chosen in order to satisfy the following conditions (see lemma 9.15 in [6]) for every $A \in$ $\mathscr{S}_{\lambda, \Lambda}$ :

(i') $1 \leq \varphi_{\eta} \leq 1+\eta h$ in $\bar{D}$, 
(ii') $\operatorname{Tr}\left(A D^{2} \varphi_{\eta}\right)-c_{1} D_{t} \varphi_{\eta}-C\left|\nabla \varphi_{\eta}\right|^{2} / \varphi_{\eta}-c_{2}\left|\nabla \varphi_{\eta}\right| \geq 0$ in $D$,

(iii') $\varphi_{\eta} \geq 1+k \eta h$ in $B_{1 / 2}^{\prime} \times\left(-\frac{T}{2}, \frac{T}{2}\right)$,

(iv') $\varphi_{\eta}=1$ in $D \backslash\left(B_{7 / 8}^{\prime} \times\left(-\frac{7 T}{8}, \frac{7 T}{8}\right)\right)$,

(v') $D_{t} \varphi_{\eta},\left|\nabla \varphi_{\eta}\right| \leq \bar{C} \eta h$ in $\bar{D}$,

(vi') $D_{t} \varphi_{\eta} \geq 0$ in $D$.

We compute

$$
\begin{aligned}
\nabla \varphi_{\eta}=\frac{1}{1-C}(1+ & \left.\psi_{\eta}\right)^{\frac{C}{1-C}} \nabla \psi_{\eta}, \quad D_{t} \varphi_{\eta}=\frac{1}{1-C}\left(1+\psi_{\eta}\right)^{\frac{C}{1-C}} D_{t} \psi_{\eta}, \\
\operatorname{Tr}\left(A D^{2} \varphi_{\eta}\right)= & \frac{1}{1-C}\left(\frac{C}{1-C}\right)\left(1+\psi_{\eta}\right)^{\frac{2 C-1}{1-C}} \operatorname{Tr}\left(A \nabla \psi_{\eta} \otimes \nabla \psi_{\eta}\right) \\
& +\frac{1}{1-C}\left(1+\psi_{\eta}\right)^{\frac{C}{1-C}} \operatorname{Tr}\left(A D^{2} \psi_{\eta}\right) .
\end{aligned}
$$

Thus, we first look for $\eta$ such that $\psi_{\eta} \leq 0$ and

$$
\begin{aligned}
& \operatorname{Tr}\left(A D^{2} \varphi_{\eta}\right)-C \frac{\left|\nabla \varphi_{\eta}\right|^{2}}{\varphi_{\eta}}-c_{2}\left|\nabla \varphi_{\eta}\right|-c_{1} D_{t} \psi_{\eta} \\
& =\frac{1}{1-C}\left(1+\psi_{\eta}\right)^{\frac{C}{1-C}}\left\{\operatorname{Tr}\left(A D^{2} \psi_{\eta}\right)\right. \\
& \left.+\left(\frac{C}{1-C}\right)\left(1+\psi_{\eta}\right)^{\frac{2 C-1}{1-C}} \operatorname{Tr}\left(A \nabla \psi_{\eta} \otimes \nabla \psi_{\eta}\right)\right\} \\
& -\frac{1}{1-C}\left(1+\psi_{\eta}\right)^{\frac{C}{1-C}}\left\{\frac{\frac{C}{(1-C)}\left(1+\psi_{\eta}\right)^{\frac{C}{1-C}}\left|\nabla \psi_{\eta}\right|^{2}}{\left(1+\psi_{\eta}\right)^{\frac{1}{1-C}}}+c_{1} D_{t} \psi_{\eta}\right\} \\
& -\frac{c_{2}}{1-C}\left(1+\psi_{\eta}\right)^{\frac{1}{1-C}-1}\left|\nabla \psi_{\eta}\right| \geq 0 .
\end{aligned}
$$

Since $C>1$, it is enough that $\psi_{\eta}$ satisfies the inequality

$$
\operatorname{Tr}\left(A D^{2} \psi_{\eta}\right)-c_{1} D_{t} \psi_{\eta}-c_{2}\left|\nabla \psi_{\eta}\right| \leq 0,
$$

where $c_{2}$ and $c_{1}$ are positive constants.

Set $x_{0}=\frac{3}{4} e_{n}$, and consider the function

$$
{ }_{0}(p)=-E\left|x-x_{0}\right|^{-N}-t,
$$

where $E$ and $N$ are positive constants to be chosen. We have

$$
\nabla \psi_{0}=N E\left|x-x_{0}\right|^{-N-1} \frac{x-x_{0}}{\left|x-x_{0}\right|}=N E\left|x-x_{0}\right|^{-N-2}\left(x-x_{0}\right)
$$

and

$$
\begin{aligned}
D_{i j} \psi_{0}= & -N(N+2) E\left|x-x_{0}\right|^{-N-4}\left(x_{i}-x_{i 0}\right)\left(x_{j}-x_{j 0}\right) \\
& +N E\left|x-x_{0}\right|^{-N-2} \delta_{i j} .
\end{aligned}
$$


Hence

$$
D^{2} \psi_{0}=-N(N+2) E\left|x-x_{0}\right|^{-N-2} v \otimes v+N E\left|x-x_{0}\right|^{-N-2} I .
$$

Thus

$$
\begin{aligned}
\operatorname{Tr}\left(A D^{2} \psi_{0}\right)= & -N(N+2) E\left|x-x_{0}\right|^{-N-2} \sum_{i, j=1}^{n} a_{i j} v_{i} v_{j} \\
& +\operatorname{Tr}(A) N E\left|x-x_{0}\right|^{-N-2} \\
\leq & N E\left|x-x_{0}\right|^{-N-2}(-(N+2) \lambda+n \Lambda)
\end{aligned}
$$

On the other hand, $D_{t} \psi_{0}=-1<0$. Set $\widetilde{\psi}_{\eta}=\eta h \psi_{0}$. If $E>T$, then $\widetilde{\psi}_{\eta} \leq 0$ in $D$. Moreover,

$$
\begin{aligned}
\operatorname{Tr}\left(A D^{2} \widetilde{\psi}_{\eta}\right)-c_{1} D_{t} \tilde{\psi}_{\eta} & \leq \eta h\left(N E\left|x-x_{0}\right|^{-N-2}(-(N+2) \lambda+n \Lambda)+c_{1}\right) \\
& \leq \eta h\left(N E 2^{-N-2}(-(N+2) \lambda+n \Lambda)+c_{1}\right) \leq 0
\end{aligned}
$$

whenever $N \geq n \frac{\Lambda}{\lambda}-2$ and

$$
0<c_{1}<N E 2^{-N-2}((N+2) \lambda-n \Lambda) .
$$

Clearly $\left|\widetilde{\psi}_{\eta}\right|$ and $|\nabla \widetilde{\psi}|$ are small with $h$.

Let now $m_{0}$ be a small positive constant such that $\widetilde{\psi}_{\eta}+m_{0} \geq 0$ in $D \backslash\left(B_{7 / 8}^{\prime} \times\right.$ $\left(-\frac{6 T}{7}, \frac{6 T}{7}\right)$ and set

$$
\widehat{\psi}_{\eta}=\min \left\{m_{0}+\tilde{\psi}_{\eta}, 0\right\} .
$$

After a mollification we get a function $\psi_{\eta}$ that satisfies the following properties for small $c_{1}$ and $c_{2}$ and $a, b, \tilde{c}$, and $h$ as in lemma 11 in [4]:

- $-a \eta \leq \psi_{\eta} \leq 0$ in $\bar{D}$,

- $\operatorname{Tr}\left(A D^{2} \psi_{\eta}\right)-c_{1} D_{t} \psi_{\eta}+C\left|\nabla \psi_{\eta}\right|^{2} / \psi_{\eta}-c_{2}\left|\nabla \psi_{\eta}\right| \leq 0$ in $D$,

- $\quad \eta=0$ in $\left.D \backslash B_{8 / 9}^{\prime} \times\left(-\frac{7 T}{8}, \frac{7 T}{8}\right)\right)$,

- $\eta \leq-k \eta b h$ in $B_{1 / 2}^{\prime} \times\left[-\frac{T}{2}, \frac{T}{2}\right]$,

- $\left|D_{t} \psi_{\eta}\right|,\left|\nabla \psi_{\eta}\right| \leq \tilde{c} \eta h$, in $D$,

- $D_{t} \psi_{\eta} \leq 0$ in $D$.

Then the function

$$
\varphi_{\eta}=\left(1+\psi_{\eta}\right)^{\frac{1}{1-C}}
$$

satisfies all the requirements $\left(\mathrm{i}^{\prime}\right)-\left(\mathrm{v}^{\prime}\right)$.

Step 2. Define

$$
\phi_{\eta}=\varphi_{\eta}+\omega\left(|x|^{2}-1\right) .
$$

Then

$$
\left|\nabla \phi_{\eta}\right| \leq\left|\nabla \varphi_{\eta}\right|+2 \omega \leq \bar{C} \eta h+2 \omega .
$$


Now, for every matrix $A$ we can write, using the properties of $\varphi_{\eta}$,

$$
\begin{aligned}
\operatorname{Tr}( & \left.A D^{2} \phi_{\eta}\right)-c_{1} D_{t} \phi_{\eta}-C \frac{\left|\nabla \phi_{\eta}\right|^{2}+\omega^{2}}{\phi_{\eta}}-c_{2}\left(\left|\nabla \phi_{\eta}\right|+\omega\right) \\
= & \operatorname{Tr}\left(A D^{2} \varphi_{\eta}\right)-c_{2} D_{t} \varphi_{\eta}+2 \omega \operatorname{Tr}(A)-C \frac{\left|\nabla \varphi_{\eta}\right|^{2}+4 \omega\left\langle\nabla \varphi_{\eta}, x\right\rangle+4 \omega^{2}|x|^{2}}{\phi_{\eta}} \\
& -c_{1}\left(\left|\nabla \varphi_{\eta}+2 \omega x\right|+\omega\right) \\
\geq & \operatorname{Tr}\left(A D^{2} \varphi_{\eta}\right)-c_{2} D_{t} \varphi_{\eta}-C \frac{\left|\nabla \varphi_{\eta}\right|^{2}}{\varphi_{\eta}}-c_{1}\left|\nabla \varphi_{\eta}\right|+2 \omega \operatorname{Tr}(A)-3 c_{1} \omega \\
& -c^{\prime \prime} \omega\left(\left|\nabla \varphi_{\eta}\right|+4 \omega\right) \\
\geq & 2 \omega \operatorname{Tr}(A)-3 c_{1} \omega-c^{\prime \prime} \omega\left(\left|\nabla \varphi_{\eta}\right|+4 \omega\right) \geq \omega\left(2 n \lambda-3 c_{1}-c^{\prime \prime}\left(\widetilde{c}_{1} \eta h+4 \omega\right)\right) .
\end{aligned}
$$

Thus, if $c_{1}$ and $\omega$ are small enough, $\phi_{\eta}$ fulfills all the properties (i)-(vi).

\subsection{Asymptotic Developments}

We now examine the asymptotic behavior at regular points on the free boundary. The following two lemmas can be proved using the arguments in lemma 13.19 and theorem 7.1 in [6].

We recall that a point $\left(x_{0}, t_{0}\right) \in F(u)$ is a regular point from the right (respectively, left) if it has a touching ball $B \subset \Omega^{+}(u)$ (respectively, $\subset \Omega^{-}(u)$ ) with tangent plane at $\left(x_{0}, t_{0}\right)$, say $\alpha^{+}\left\langle\left(x-x_{0}, v\right)\right\rangle+\beta^{+}\left(t-t_{0}\right)=0$.

LEMMA 3.4. Let $u$ be a viscosity solution in $\mathscr{C}_{1,1}$ of our f.b.p. satisfying the hypotheses of the main Theorem 1.2. Suppose $(0,0) \in F(u)$. Then, near $(0,0)$, the following asymptotic inequalities hold:

(i) If $(0,0)$ is regular from the right,

$$
\begin{gathered}
u(x, t) \geq\left(\beta^{+} t+\alpha^{+}\langle x, v\rangle\right)^{+}-\left(\beta^{-} t+\alpha^{-}\langle x, v\rangle\right)^{-}+o\left(d_{x, t}\right) \\
\text { with } \alpha^{+}>0, \alpha^{-} \geq 0, \beta^{+}, \beta^{-} \in \mathbb{R}, \text { and } \\
\beta^{+} \geq \alpha^{+} G\left(\alpha^{+}, \alpha^{-}\right) .
\end{gathered}
$$

(ii) If $(0,0)$ is regular from the left,

$$
\begin{gathered}
u(x, t) \leq\left(\beta^{+} t+\alpha^{+}\langle x, v\rangle\right)^{+}-\left(\beta^{-} t+\alpha^{-}\langle x, v\rangle\right)^{-}+o\left(d_{x, t}\right) \\
\text { with } \alpha^{+} \geq 0, \alpha^{-}>0, \beta^{+}, \beta^{-} \in \mathbb{R}, \text { and } \\
\beta^{+} \leq \alpha^{+} G\left(\alpha^{+}, \alpha^{-}\right) .
\end{gathered}
$$

Moreover, equality holds in the above asymptotic expansions along paraboloids of the form $t=-\gamma\langle x, v\rangle^{2}, \gamma>0$.

For the next lemma, recall that the solution of our f.b.p. enjoys the monotonicity properties expressed in Lemmas 2.1 and 2.2 along the spatial cone $\Gamma^{x}\left(e_{n}, \theta\right)$ and the space-time cone $\Gamma^{t}\left(e_{n}, \theta^{t}\right)$. 
LEMMA 3.5. Let $u$ be a viscosity solution of a free boundary problem satisfying the hypotheses of Theorem 1.2 Let

$$
\bar{v}_{\eta}(p)=\sup _{B_{\phi_{\eta}}(p)} u
$$

where $\phi_{\eta}$ is as in Lemma 3.3

Suppose the supremum in (3.5) occurs uniformly away from the top and the bottom points of the ball, at a distance not smaller than some fixed constant $\rho$. Then the following hold:

(i) $\partial \Omega^{+}\left(v_{\eta}\right)$ is uniformly Lipschitz in space-time with Lipschitz constant in space bounded above by $\tan \left(\frac{\pi}{2}-\theta\right)+c\left|\nabla \phi_{\eta}\right|$.

(ii) If $\left(x_{0}, t_{0}\right) \in F\left(v_{\eta}\right)$ and $\left(y_{0}, s_{0}\right) \in F(u)$ with

$$
\left(y_{0}, t_{0}\right) \in \partial B_{\phi_{\eta}\left(x_{0}, t_{0}\right)}\left(x_{0}, t_{0}\right),
$$

then $\left(x_{0}, t_{0}\right)$ is a regular point from the right. Moreover, if near $\left(y_{0}, s_{0}\right)$ along the paraboloid $s=s_{0}-\gamma\left\langle y-y_{0}, v\right\rangle^{2}(\gamma>0)$, $u$ has the asymptotic expansion

$$
u(q)=\alpha^{+}\left\langle y-y_{0}, v\right\rangle^{+}-\alpha^{-}\left\langle y-y_{0}, v\right\rangle^{-}+o\left(\left|y-y_{0}\right|\right)
$$

where $v=\left(y_{0}-x_{0}\right) /\left|y_{0}-x_{0}\right|$, then near $\left(x_{0}, t_{0}\right)$ along the paraboloid $t=t_{0}-\gamma\left\langle x-x_{0}, v\right\rangle^{2}$

$$
\begin{aligned}
& \bar{v}_{\eta}(p) \geq \alpha^{+}\left\langle x-x_{0}, v+\frac{\phi_{\eta}\left(x_{0}, t_{0}\right)}{\left|y_{0}-x_{0}\right|} \nabla\left(\phi_{\eta}\right)\right\rangle^{+} \\
& -\alpha^{-}\left\langle x-x_{0}, v+\frac{\phi_{\eta}\left(x_{0}, t_{0}\right)}{\left|y_{0}-x_{0}\right|} \nabla\left(\phi_{\eta}\right)\right\rangle^{-}+o\left(\left|x-x_{0}\right|\right), \\
& \text { with } \frac{s_{0}-t_{0}}{\left|y_{0}-x_{0}\right|} \leq G\left(\alpha^{+}, \alpha^{-}\right) \text {. }
\end{aligned}
$$

Remark 3.6. A similar lemma holds with reverse inequalities for

$$
\underline{v}_{\eta}(p)=\inf _{B_{\phi_{\eta}}(p)} u
$$

\section{Local Stability Results for the Free Boundary}

In this section we prove that under a small perturbation of the coefficients of our operator, the free boundary undergoes a small perturbation too. For technical reasons it is more convenient to work in a different kind of space-time cylinder. Thus let

Note that $\mathscr{C}_{1}^{*} \subset B_{\sqrt{2}}^{\prime}$.

$$
\begin{aligned}
\Phi_{R, r} & =\left\{\left(x^{\prime}, x_{n}\right):\left|x^{\prime}\right|<R,\left|x_{n}\right|<r\right\}, \\
\mathscr{C}_{R, r, s,}^{*} & =\Phi_{R, r} \times(-s, s), \quad \mathscr{C}_{s}^{*}=\mathscr{C}_{s, s, s}^{*} .
\end{aligned}
$$

Let $u$ be a solution of our f.b.p. in $\mathscr{C}_{2}^{*}$. Observe that on $\left\{\left|x^{\prime}\right|<1, x_{n}= \pm 1\right\} \times$ $\left(-\frac{3}{2}, \frac{3}{2}\right)$ we have $u \sim u\left(p_{0}^{+}\right) \sim 1$ and $u \sim u\left(p_{0}^{-}\right) \sim m$, respectively, where $p_{0}^{ \pm}=\left( \pm \frac{1}{2} e_{n}, 0\right)$. 
Recall that $u$ is monotone along the directions $\tau \in \Gamma^{x}\left(e_{n}, \theta\right)$ and $\epsilon_{0}$-monotone along $\Gamma^{t}\left(e_{n}, \theta_{t}\right)$, with $\frac{\pi}{4} \leq \theta^{t}<\frac{\pi}{2}$. Moreover, $u$ is fully monotone along $\Gamma^{t}\left(e_{n}, \theta_{t}\right)$ outside a $\epsilon_{0}$-neighborhood of $F(u)$. Let $\phi_{0}(x, t)=1+\omega\left(|x|^{2}-2\right)$ with $\omega=\omega(1) \ll \delta=\frac{\pi}{2}-\theta$ and also $\omega \leq \epsilon_{0}^{(1-\alpha) / \alpha}$. We assume that $\|A-B\|_{\infty} \leq \omega$ and $\left\|\mathbf{b}-\mathbf{b}^{\prime}\right\|_{\infty} \leq \omega$ (see (3.1) and Remark 3.2).

Note that $\phi_{0}$ is one of the functions constructed in Lemma 3.3, corresponding to the parameter $\eta=0$. Clearly $\max _{\bar{B}_{\sqrt{2}}^{\prime} \times \mathbb{R}} \phi_{0}=1$.

THEOREM 4.1. Let $z$ be the solution of our f.b.p. for the heat operator in $\mathscr{C}_{1}^{*}$ with $z=u$ on the parabolic boundary $\partial_{p} \mathscr{C}_{1}^{*}$. Then:

(a) $F(z) \cap \mathscr{C}_{1}^{*}$ is contained in a $\epsilon_{0}$-neighborhood $\mathscr{N}_{\epsilon_{0}}(F(u))$ of $F(u)$.

(b) $z$ is $\epsilon_{0}$-monotone along the directions $\tau \in \Gamma^{x}\left(e_{n}, \theta^{\prime}\right) \cup \Gamma^{t}\left(e_{n}, \theta_{t}^{\prime}\right)$, with $\theta^{\prime}$ and $\theta_{t}^{\prime}$ slightly smaller than $\theta$ and $\theta_{t}$, respectively (to be specified later).

(c) If $\epsilon_{0}$ and $\delta^{\prime}=\frac{\pi}{2}-\theta^{\prime}$ are small enough, depending only on $n$ and $\theta_{t}^{\prime}$, in $\mathscr{C}_{1 / 2}^{*}$, then $F(z)$ is the graph of a $C^{1}$-function $f^{*}=f^{*}\left(x^{\prime}, t\right)$. In particular, $z$ is a classical solution and

$$
\begin{aligned}
z(p) & \geq C d_{p} \quad \text { in } \mathscr{C}_{1 / 2}^{*}, \\
|\nabla z(p)| & \geq C_{0} \quad \text { in } \mathscr{C}_{1 / 2}^{*} \cap F(z),
\end{aligned}
$$

where $p=(x, t)$.

PROOF. We split the proof into several steps.

Step 1 . We first show that $F(z)$ stays at most $\epsilon_{0}$ away to the left of $F(u)$.

Choose $\theta^{\prime}$ and $\theta_{t}^{\prime}$ slightly smaller than $\theta$ and $\theta_{t}$, respectively, such that, if $\tau$ is a vector belonging to $\Gamma^{x}\left(e_{n}, \theta^{\prime}-\delta\right) \cup \Gamma^{t}\left(e_{n}, \theta_{t}^{\prime}-\delta\right)$ with $\epsilon_{0}=|\tau| \sin (\delta / 2)$ in $\mathscr{C}_{3 / 2}^{*}$ we can write

$$
\bar{v}_{0}(p) \equiv \sup _{q \in B_{\epsilon_{0} \phi_{0}}(p)} u(q-\tau) \leq u(p)
$$

with the free boundary of $\bar{v}_{0} \epsilon_{0}$-away from $F(u)$. We know that $\bar{v}_{0}$ is an $\mathscr{L}$ subsolution in the complement of its zero set. Since $D_{\tau /|\tau|} u \geq c \delta D_{n} u$, uniformly in $\Omega^{+}\left(\bar{v}_{0}\right) \cap \mathscr{C}_{3 / 2}^{*}$, we get

$$
\bar{v}_{0}(p)<\left(1-c \epsilon_{0}\right) u(p)
$$

in the same set. Indeed, if

$$
\bar{v}_{0}(p)=u(\bar{q}-\tau)
$$

we have, using (2.4),

$$
\begin{aligned}
u(p)-\bar{v}_{0}(p)=u(p)-u(\bar{q}-\tau) & \geq c_{1} \delta|p-\bar{q}+\tau| D_{n} u(p) \\
& \geq c_{2} \delta|| \tau\left|-\epsilon_{0}\right| u(p)>c \epsilon_{0} u(p) .
\end{aligned}
$$

Let $w^{+}$be the caloric measure in $\Omega^{+}\left(\bar{v}_{0}\right) \cap \mathscr{C}_{1+\bar{\epsilon}, 1,1+\bar{\epsilon}}^{*}$ of

$$
\Sigma^{+}=\left\{\left|x^{\prime}\right|<1+\bar{\epsilon}, x_{n}=1\right\} \times(-1-\bar{\epsilon}, 1+\bar{\epsilon}),
$$


extended by zero outside $\Omega^{+}\left(\bar{v}_{0}\right)$. From (4.1), if $\bar{\epsilon}$ is properly chosen, depending only on the Harnack constant for $u$ and the flatness of $F(u)$, we get

$$
\bar{V}(p) \equiv \bar{v}_{0}(p)+c^{\prime} \epsilon_{0} u\left(p_{0}^{+}\right) w^{+}(p)<z(p)
$$

on $\partial_{p} \mathscr{C}_{1}^{*}$, since $z=u$ there.

We want to show that $\bar{V}$ is a viscosity subsolution of the two-phase problem in $\mathscr{C}_{1}^{*}$. We know from Theorem 3.1 that $\bar{V}$ is subcaloric in both $\Omega^{ \pm}(\bar{V})$. We have only to check the free boundary condition.

Thus, let $Q \subset \mathscr{C}_{1}^{*}$ be a cylinder and $\varphi$ be a classical supersolution of the twophase problem such that $\varphi \geq \bar{V}$ on $\partial_{p} Q$.

It is actually enough to consider those $\varphi$ such that $\varphi>\bar{V}$ on $\partial_{p} Q$. Indeed, if this is true, let $\varphi \geq \bar{V}$ on $\partial_{p} Q$. Since both $\bar{v}_{0}(p)$ and $w^{+}$are strictly increasing along $e_{n}$ (for $w^{+}$compare with translations), then $\bar{V}$ is strictly increasing along $e_{n}$. Hence, for a small positive $\sigma>0$, we can write $\varphi(p)>\bar{V}\left(p-\sigma e_{n}\right)$ on $\partial_{p} Q$, that is, $\tilde{\varphi}(p)=\varphi\left(p+\sigma e_{n}\right)>\bar{V}(p)$ on $\partial_{p} Q-\sigma e_{n}$, and we deduce $\tilde{\varphi}(p)>\bar{V}(p)$ on $Q-\sigma e_{n}$. Letting $\sigma \rightarrow 0$ we get $\varphi \geq \bar{V}$ on $Q$.

We now argue by contradiction. Suppose that $t_{0}$ is the first time at which the two free boundaries touch each other. If $\left(x_{0}, t_{0}\right) \in F(\bar{V}) \cap F(\varphi)$, then $\left(x_{0}, t_{0}\right)$ is regular from both sides. If $\left(y_{0}, s_{0}\right)$ is the point where $u_{1}(q) \equiv u(q-\tau)$ achieves its maximum in the ball $B_{\epsilon_{0} \phi_{0}}(p)$, then the interior normal is

$$
v=\frac{v^{*}}{\left|v^{*}\right|}, \quad v^{*}=\frac{y_{0}-x_{0}+\epsilon_{0}^{2} \phi_{0}\left(x_{0}, t_{0}\right) \nabla_{x} \phi_{0}\left(x_{0}, t_{0}\right)}{\left|x_{0}-y_{0}\right|} .
$$

Set $\beta^{+}=D_{t} \varphi^{+}\left(x_{0}, t_{0}\right)$ and $\alpha^{ \pm}=\left|\nabla \varphi^{ \pm}\left(x_{0}, t_{0}\right)\right|$. We have

$$
\frac{\beta^{+}}{\alpha^{+}} \geq G\left(\alpha^{+}, \alpha^{-}\right) \text {. }
$$

From (i) of Lemma 3.5 and (v) of Lemma 3.3 , we get that $F(\bar{V})$ is a Lipschitz surface with Lipschitz constant controlled by $(1+\bar{\eta}) \delta$, where $\bar{\eta}$ is a small number. Thus, since by construction $\left(x_{0}, t_{0}\right)$ is $\bar{\epsilon}$-away from $\partial_{p} \mathscr{C}_{1,1, \bar{\epsilon}}$, we can use the contact point Lemma 5.1 in [5] and deduce

$$
D_{\nu} w^{+}\left(x_{0}, t_{0}\right) \geq c^{*}(\bar{\epsilon})>0 .
$$

Now, near $\left(y_{0}, s_{0}\right)$ along the paraboloid $s=s_{0}-\gamma\left\langle y-y_{0}, v_{1}\right\rangle^{2}$, where $v_{1}=$ $\left(y_{0}-x_{0}\right) /\left|y_{0}-x_{0}\right|, u_{1}$ has the asymptotic development

$$
u_{1}(y, s)=\alpha_{1}^{+}\left\langle y-y_{0}, v_{1}\right\rangle^{+}-\alpha_{1}^{-}\left\langle y-y_{0}, v_{1}\right\rangle^{-}+o\left(\left|y-y_{0}\right|\right)
$$

with

$$
\frac{s_{0}-t_{0}}{\left|y_{0}-x_{0}\right|} \leq G\left(\alpha_{1}^{+}, \alpha_{1}^{-}\right) .
$$

By the boundary Harnack principle we have, near $\left(x_{0}, t_{0}\right)$,

$$
w^{+} \geq \widetilde{c}(\bar{\epsilon}) \bar{V} .
$$


Thus near $\left(x_{0}, t_{0}\right)$, by Lemma 3.5, we can write

$$
\bar{V}(x, t) \geq \hat{\alpha}^{+}\left\langle x-x_{0}, v\right\rangle^{+}-\alpha_{1}^{-}\left\langle x-x_{0}, v\right\rangle^{-}+o\left(\left|x-x_{0}\right|\right)
$$

where

$$
\hat{\alpha}^{+} \geq \alpha_{1}^{+}+C c^{*} \epsilon_{0}
$$

if $\left|\nabla \phi_{0}\right| \leq \omega$ is kept small.

Then, we have (see [5, lemma 8.3]), by (4.2) and the Lipschitz continuity of $G$,

$$
\begin{aligned}
G\left(\alpha^{+}, \alpha^{-}\right) & \leq \frac{\beta^{+}}{\alpha^{+}}=\frac{s_{0}-t_{0}}{\left|y_{0}-x_{0}\right|}\left|\nu^{*}\right|^{-1} \\
& \leq \frac{s_{0}-t_{0}}{\left|y_{0}-x_{0}\right|}\left(1+c_{1} \epsilon_{0} \omega\right) \\
& \leq G\left(\alpha_{1}^{+}, \alpha_{1}^{-}\right)+c_{2} \epsilon_{0} \omega \\
& \leq G\left(\hat{\alpha}_{1}^{+}, \alpha_{1}^{-}\right)-\epsilon_{0}\left(C c^{*}-C_{2} \omega\right) \\
& <G\left(\hat{\alpha}_{1}^{+}, \alpha_{1}^{-}\right)
\end{aligned}
$$

as long as $\omega<C c^{*} / C_{2}$.

By the strict monotonicity of $G$ and the Hopf maximum principle, we reach a contradiction, since $\alpha^{+}>\hat{\alpha}_{1}^{+}$and $\alpha^{-}<\alpha_{1}^{-}$. We conclude that $\bar{V}$ is a viscosity subsolution of the f.b.p.

Theorem 3.1 in [14] now gives

$$
\bar{V}<z \quad \text { in } \mathcal{C}_{1}^{*} .
$$

This inequality says that $F(z)$ stays at most $\epsilon_{0}$-away to the left of $F(u)$.

To show that $F(z)$ stays at most $\epsilon_{0}$-away to the right of $F(u)$, the argument is the same. We know that

$$
\underline{v}_{0}(p) \equiv \inf _{q \in B_{\epsilon_{0} \phi_{0}}(p)} u(q+\tau) \geq u(p)
$$

in $\mathscr{C}_{3 / 2}^{*}$ and

$$
\underline{v}_{0}(p)>\left(1-c \epsilon_{0} \delta\right) u(p)
$$

in $\Omega^{-}\left(\underline{v}_{0}\right) \cap \mathscr{C}_{3 / 2}^{*}$. Moreover, $\underline{v}_{0}(p)$ is an $\mathscr{L}$-supersolution in the complement of its zero set; see Remark 3.6 .

Now let $w^{-}$be the caloric measure in $\Omega^{-}\left(\underline{v}_{0}\right) \cap \mathscr{C}_{1+\bar{\epsilon}, 1,1+\bar{\epsilon}}^{*}$ of

$$
\Sigma^{-}=\left\{\left|x^{\prime}\right|<1+\bar{\epsilon}, x_{n}=-1\right\} \times(-1-\bar{\epsilon}, 1+\bar{\epsilon}),
$$

extended by zero outside $\Omega^{-}\left(\underline{v}_{0}\right)$. Then we can write

$$
\underline{V}(p) \equiv \underline{v}_{0}(p)-c^{\prime} \epsilon_{0} u\left(p_{0}^{-}\right) w^{-}>z(p)
$$

on $\partial_{p} \mathscr{C}_{1}^{*}$, since $z=u$ there. 
As before we can show that $\underline{V}$ is a viscosity supersolution of the two-phase problem in $\mathscr{C}_{1}^{*}$. This time, theorem 3.1 in [14] gives

$$
\underline{V}>z \quad \text { in } \mathscr{C}_{1}^{*} \text {. }
$$

This inequality says that $F(z)$ stays at most $\epsilon_{0}$-away to the right of $F(u)$ and (a) follows.

Step 2. The results in part (a) are valid for any $\epsilon^{\prime}$ such that $\epsilon_{0} \leq \epsilon^{\prime} \leq 2 \epsilon_{0}$. Thus we have, for every vector $\tau \in \Gamma^{x}\left(e_{n}, \hat{\theta}-\delta\right) \cup \Gamma^{t}\left(e_{n}, \hat{\theta}^{t}-\delta\right)$ such that $\epsilon^{\prime}=|\tau| \sin \left(\frac{\delta}{2}\right)$,

$$
z(p) \leq \inf _{q \in B_{\epsilon^{\prime} \phi_{0}}(p)} u(q+\tau) \leq u(p+\tau) \leq \sup _{q \in B_{\epsilon^{\prime} \phi_{0}}(p+\tau)} u(q) \leq z(p+\tau) .
$$

It is now easy to deduce that $z$ is $\epsilon_{0}$-monotone along the selected directions.

Step 3. From (b), $z$ falls under the hypotheses of theorem 1.4 in [5] and the conclusion follows.

Conclusion (a) of Theorem 4.1 can be extended to solutions of our f.b.p. governed by more general operators

$$
\tilde{\mathscr{L}}=\operatorname{Tr}\left(\tilde{A} D^{2}\right)+\tilde{\mathbf{b}} \cdot \nabla
$$

belonging to the class $\mathscr{A}$. Specifically, we have the following, taking for simplicity $\widetilde{\mathbf{b}}=\mathbf{0}$ :

THEOREM 4.2. Assume the hypotheses of Theorem 1.2 hold. Let $\tilde{\mathscr{L}}$ be another operator in the class $\mathscr{A}$, and let $\tilde{u}$ be a solution of our f.b.p. in $\mathscr{C}_{1}^{*}$ for the operator $\tilde{\mathscr{L}}$, with $\tilde{u}=u$ on $\partial_{p} \mathscr{C}_{1}^{*}$. Then, if $\|A-\tilde{A}\|_{\infty} \leq \omega$ and $\epsilon_{0}$ and $\omega$ are as in Theorem 4.1 $F(\tilde{u}) \cap \mathscr{C}_{1}^{*}$ is contained in a $\epsilon_{0}$-neighborhood of $F(u)$.

PROOF. We follow the proof of Theorem 4.1, keeping also the same notation, until we get inequality 4.1 :

$$
\bar{v}_{0}(p)<\left(1-c \epsilon_{0}\right) u(p)
$$

valid in $\Omega^{+}\left(\bar{v}_{0}\right) \cap \mathscr{C}_{3 / 2}^{*}$.

Set $\Omega_{\bar{\epsilon}}^{+}\left(\bar{v}_{0}\right)=\Omega^{+}\left(\bar{v}_{0}\right) \cap \mathscr{C}_{1+\bar{\epsilon}, 1,1+\bar{\epsilon}}^{*}$ and, as before,

$$
\Sigma^{+}=\left\{\left|x^{\prime}\right|<1+\bar{\epsilon}, x_{n}=1\right\} \times(-1-\bar{\epsilon}, 1+\bar{\epsilon}) .
$$

Denote by $\mathscr{P}^{-}$the extremal Pucci operator

$$
\mathscr{P}^{-}(v)=\inf _{M \in \mathscr{S}_{\lambda, \Lambda}} \operatorname{Tr}\left(M D^{2} v\right) .
$$

Let $\mathbb{1}_{\Sigma^{+}}$be the characteristic function of $\Sigma^{+}$and $W^{+}$be the solution of the problem

$$
\begin{cases}\mathscr{P}^{-}\left(W^{+}\right)-D_{t} W^{+}=0 & \text { in } \Omega_{\bar{\epsilon}}^{+}\left(\bar{v}_{0}\right) \\ W^{+}=\mathbb{1}_{\Sigma^{+}} & \text {on } \partial_{p} \Omega_{\bar{\epsilon}}^{+}\left(\bar{v}_{0}\right)\end{cases}
$$


extended by zero outside $\Omega^{+}\left(v_{0}^{+}\right)$. Note that, comparing with translations, we get that $W^{+}$is increasing along the $e_{n}$-direction. Define

$$
\bar{Z}(p) \equiv \bar{v}_{0}(p)+c^{\prime} \epsilon_{0} u\left(p_{0}^{+}\right) W^{+}(p) .
$$

As in the proof of Theorem 4.1 , we know that

$$
\bar{Z}(p)<\tilde{u}(p)
$$

on $\partial_{p} \mathscr{C}_{1}^{*}$ since $\tilde{u}=u$ there.

We want to show that $\bar{Z}$ is a viscosity subsolution in $\mathscr{C}_{1}^{*}$ for each operator $\tilde{\mathscr{L}} \in \mathscr{A}$, satisfying the hypotheses of Theorem 4.2 . Notice that $\bar{Z}$ is $\tilde{\mathscr{L}}$-subcaloric in both $\Omega^{ \pm}(\bar{Z})$.

Again we have only to check the free boundary condition. Thus, let $Q \subset \mathscr{C}_{1}^{*}$ be a cylinder and $\varphi$ be a classical supersolution such that $\varphi \geq \bar{Z}$ on $\partial_{p} Q$. It is enough to consider those $\varphi$ such that $\varphi>\bar{Z}$ on $\partial_{p} Q$ and to show that $\varphi>\bar{Z}$ in $Q$. Indeed, let $\varphi \geq \bar{Z}$ on $\partial_{p} Q$.

Choose a small positive $\sigma, \sigma \ll \omega$. We have $\bar{Z}\left(p-\sigma e_{n}\right)<\bar{Z}(p)$ on $\partial_{p} Q$, since $\bar{Z}$ is strictly increasing along $e_{n}$. Thus $\tilde{\varphi}(p)=\varphi\left(p+\sigma e_{n}\right)>\bar{Z}(p)$ on the shifted parabolic boundary $\partial_{p} Q-\sigma e_{n}$. Since

$$
\tilde{\mathscr{L}}_{\sigma}=\sum_{i, j=1}^{n} \tilde{a}_{i j}\left(p+\sigma e_{n}\right) D_{i j}
$$

satisfies the hypotheses of Theorem 4.2 , we deduce that $\widetilde{\varphi}(p)>\bar{Z}(p)$ in $Q-\sigma e_{n}$. Letting $\sigma \rightarrow 0$, we deduce $\varphi(p) \geq Z(p)$ in $Q$.

From now on we can proceed as in the proof of Theorem 4.1, using the Pucci operator

$$
\mathscr{P}^{+}(v)=\sup _{M \in \mathscr{S}_{\lambda, \Lambda}} \operatorname{Tr}\left(M D^{2} v\right)
$$

and modifying accordingly the arguments for

$$
\underline{Z}(p)=\underline{v}_{0}(p)-c^{\prime} \epsilon_{0} u\left(p_{0}^{-}\right) W^{-}
$$

where $W^{-}$is the solution of

$$
\begin{cases}\mathscr{P}^{+}\left(W^{-}\right)-D_{t} W^{-}=0 & \text { in } \Omega_{\epsilon}^{-}\left(\underline{v}_{0}\right) \\ W^{-}=\mathbb{1}_{\Sigma^{-}} & \text {on } \partial_{p} \Omega_{\epsilon}^{-}\left(\underline{v}_{0}\right) .\end{cases}
$$

In conclusion, it turns out that

$$
\bar{Z}(p)<\tilde{u}(p)<\underline{Z}(p)
$$

in $\mathscr{C}_{1}^{*}$, which implies, in particular, that $F(\widetilde{u})$ is contained in an $\epsilon_{0}$-neighborhood of $F(u)$.

We now want to give estimates for $u, \nabla u$, and $D_{t} u$ in terms of $z, \nabla z$, and $D_{t} z$. 
LEMMA 4.3. Let $\widetilde{\Omega}=\mathcal{C}_{1}^{*} \cap \Omega^{+}(u) \cap \Omega^{+}(z)$. Under the stated hypotheses, on $\partial_{p} \widetilde{\Omega}$ we have

$$
|u-z| \leq c_{0} \epsilon_{0}^{a} u\left(p_{0}^{+}\right)
$$

where $a$ is the Hölder exponent of $u$ across the free boundary.

PROOF. For any point $p \in F(u) \cap \Omega^{+}(z)$ with distance of order $\epsilon_{0}$ from $\partial_{p} \mathcal{C}_{1}^{*}$, by the monotonicity properties of $z$, the Carleson estimate, and the maximum principle, we have the following:

$$
z(p) \leq c \epsilon_{0} z\left(p_{0}^{+}\right) \leq c \epsilon_{0} u\left(p_{0}^{+}\right) .
$$

For the remaining points $p$ on $F(u) \cap \Omega^{+}(z)$ at distance less than $\epsilon_{0}$, we have, using the monotonicity of $z$ and by a Carleson estimate, if $p+\epsilon_{0} \tau \in \partial_{p} \mathcal{C}_{1}^{*}$,

$$
z(p) \leq z\left(p+\epsilon_{0} \tau\right)=u\left(p+\epsilon_{0} \tau\right) \leq c \epsilon_{0}^{a} u\left(p_{0}^{+}\right) .
$$

Analogously, for any point $p \in F(z) \cap \Omega^{+}(u)$ we have again by a Carleson estimate

$$
u(p) \leq c \epsilon_{0}^{a} u\left(p_{0}^{+}\right) .
$$

Since $u=z$ on the rest of $\partial_{p} \widetilde{\Omega}$, the proof is complete.

LEMMA 4.4. Let $Q$ be a cylinder contained in $\widetilde{\Omega}$ such that, say, $\operatorname{dist}(Q, \partial \widetilde{\Omega}) \geq$ $1 / 100$. Then there exists a constant $C$ such that, in $Q$ :

$$
\begin{aligned}
|u(p)-z(p)| & \leq c_{0} \epsilon_{0}^{\bar{a}} u(p), \\
|\nabla u(p)-\nabla z(p)| & \leq C \epsilon_{0}^{\bar{a}} D_{n} u(p), \\
\left|D_{t} u(p)-D_{t} z(p)\right| & \leq C \epsilon_{0}^{\bar{a}} D_{n} u(p),
\end{aligned}
$$

where $\bar{a}=\min \{\alpha, a \alpha /(\alpha+2)\}$.

PROOF. The proof of estimate (4.6) is contained in [11, lemma 2.1]. For the reader's convenience we reproduce here the basic steps. Let $p=(y, s) \in Q$ and

$$
\Phi_{\epsilon_{0}}=\Phi_{\epsilon_{0}}(p)=B_{\epsilon_{0}}(y) \times\left(s-\epsilon_{0}^{2}, s+\epsilon_{0}^{2}\right) .
$$

Let $w=u-z$. In $\Phi_{\epsilon_{0}}(p)$ we have

$$
D_{t} w-\Delta w=\sum_{i, j=1}^{n}\left(a_{i, j}-\delta_{i j}\right) D_{i j} u-\sum_{j=1}^{n} b_{j} D_{j} u .
$$

From Aleksandrov-Bakelman-Pucci estimates, we get

$$
\begin{aligned}
\|w\|_{L^{\infty}\left(\Phi_{\epsilon_{0}}\right) \leq} & \|w\|_{L^{\infty}\left(\partial_{p} \Phi_{\epsilon_{0}}\right)} \\
& +C_{0} \epsilon_{0}^{\frac{n}{n+1}}\left\|\sum_{i, j=1}^{n}\left(a_{i, j}-\delta_{i j}\right) D_{i j} u-\sum_{j=1}^{n} b_{j} D_{j} u\right\|_{L^{n+1}\left(\Phi_{\epsilon_{0}}\right)} .
\end{aligned}
$$


We now take into account estimate (4.4) and use the techniques in [7, lemma 1], the interior Harnack inequality, and the space-time monotonicity of $u$-away from $F(u)$. We deduce that

$$
\|w\|_{L^{\infty}\left(\partial_{p} \Phi_{\epsilon_{0}}\right)} \leq c_{0} \epsilon_{0}^{\frac{a \alpha}{\alpha+2}} u(p) .
$$

Under our hypotheses,

$$
\left\|a_{i, j}-\delta_{i j}\right\|_{L^{\infty}\left(\Phi_{\epsilon_{0}}\right)} \leq c \epsilon_{0}^{\alpha} \quad \text { and } \quad\left\|b_{j}\right\|_{L^{\infty}\left(\Phi_{\epsilon_{0}}\right)} \leq \omega(1) \leq \epsilon_{0}^{(1-\alpha) / \alpha} .
$$

Then, from interior Schauder estimates, the Harnack inequality, and the space-time monotonicity of $u$, away from $F(u)$, we get

$$
\begin{aligned}
& \left\|\sum_{i, j=1}^{n}\left(a_{i, j}-\delta_{i j}\right) D_{i j} u-\sum_{j=1}^{n} b_{j} D_{j} u\right\|_{L^{n+1}\left(\Phi_{\epsilon_{0}}\right)} \\
& \leq \epsilon_{0}^{\alpha}\left\|\sum_{i, j=1}^{n} D_{i j} u\right\|_{L^{n+1}\left(\Phi_{\epsilon_{0}}\right)}+\epsilon_{0}^{(1-\alpha) / \alpha}\left\|\sum_{j=1}^{n} D_{j} u\right\|_{L^{n+1}\left(\Phi_{\epsilon_{0}}\right)} \\
& \leq c\left(\epsilon_{0}^{\alpha-2+\frac{n+2}{n+1}}+\epsilon_{0}^{\frac{1}{\alpha}-1-1+\frac{n+2}{n+1}}\right) u(p) \leq c_{1} \epsilon_{0}^{\alpha-2+\frac{n+2}{n+1}} u(p) .
\end{aligned}
$$

Thus we have

$$
\|w\|_{L^{\infty}\left(\partial_{p} \Phi_{\epsilon_{0}}\right)} \leq\left(c_{0} \epsilon_{0}^{\frac{a \alpha}{\alpha+2}}+c_{1} C_{0} \epsilon_{0}^{\alpha-2+\frac{n+2}{n+1}+\frac{n}{n+1}}\right) u(p) \leq C \epsilon_{0}^{\bar{a}} u(p) .
$$

The other estimates follow from interior Schauder estimates, the Harnack and boundary Harnack inequalities, plus estimate 2.3.

\section{Interior Gains}

As mentioned in the introduction, we need to enlarge the cone of monotonicity away from the free boundary in hyperbolic homogeneity. To do that, we use the perturbation Theorem 4.1. Indeed, for the solution $z$ defined in Theorem 4.1, we can use the results in [4, 5]. In particular, letting $\delta_{0}=\frac{\pi}{2}-\hat{\theta}$ and $\mu_{0}=\frac{\pi}{2}-$ $\hat{\theta}^{t}$, where $\hat{\theta}$ and $\hat{\theta}^{t}$ are defined in Theorem 4.1(b), from the proof of the Main Theorem in [4], we infer that there exist a sequence of spacial cones $\Gamma^{x}\left(v_{k}^{*}, \theta_{k}^{*}\right)$ and sequences $\left\{A_{k}^{*}\right\},\left\{B_{k}^{*}\right\},\left\{\delta_{k}^{*}\right\},\left\{\mu_{k}^{*}\right\}$ with the following properties: in

$$
B_{2^{-k}}^{\prime} \times\left(-C \frac{\delta_{k}^{*}}{\mu_{k}^{*}}, C \frac{\delta_{k}^{*}}{\mu_{k}^{*}}\right), \quad k \geq 0,
$$

(i) $z$ is monotone increasing along every $\tau \in \Gamma_{x}^{*}\left(v_{k}^{*}, \theta_{k}^{*}\right)$;

(ii) $z$ is monotone along the directions $e_{t}+B_{k}^{*} v_{k}^{*}$ and $-e_{t}-B_{k}^{*} v_{k}^{*}$ where

$$
0 \leq B_{k}^{*}-A_{k}^{*} \leq c_{0}^{*} \mu_{k}^{*}
$$


(iii) the sequences $\left\{\delta_{k}^{*}\right\}$ and $\left\{\mu_{k}^{*}\right\}$ satisfy the recurrence relations

$$
\begin{aligned}
& \delta_{k+1}^{*} \leq \delta_{k}^{*}-c_{1}^{*} \frac{\delta_{k}^{*}}{\mu_{k}^{*}} \\
& \mu_{k+1}^{*} \leq \mu_{k}^{*}-c_{2}^{*} \delta_{k}^{*}
\end{aligned}
$$

and $\delta_{k}^{*} \ll\left(\mu_{k}^{*}\right)^{3}$.

The constants $C, c_{1}^{*}$, and $c_{2}^{*}$ depend only on $n$ and the Lipschitz constants of the free boundary. We now choose $\hat{\theta}$ and $\hat{\theta}^{t}$ such that $\delta_{0}$ and $\mu_{0}$ are the predecessors of $\delta$ and $\mu$ in the recurrence relations (5.2) and (5.3):

$$
\begin{aligned}
& \delta=\delta_{0}-c_{1}^{*} \frac{\delta_{0}}{\mu_{0}}, \\
& \mu=\mu_{0}-c_{2}^{*} \delta_{0} .
\end{aligned}
$$

LeMma 5.1 (Interior Gain in Space). Let $u$ be our solution of the f.b.p. and $\epsilon_{0}^{\bar{a}} \ll$ $\delta$. There exists a constant $b_{0}, 0<b_{0}<1$, such that in

$$
B_{1 / 16}^{\prime}\left(\frac{1}{8} e_{n}\right) \times\left(-C \frac{\delta}{\mu}, C \frac{\delta}{\mu}\right),
$$

$u$ is monotone increasing along the directions $\tau$ belonging to a cone $\Gamma^{x}(\bar{v}, \bar{\theta})$ such that

$$
\frac{\pi}{2}-\bar{\theta} \leq b_{0}\left(\frac{\pi}{2}-\theta\right), \quad\left|\bar{v}-e_{n}\right| \leq b_{0} \delta
$$

Proof. Consider the recurrence relation for $z$. After two iteration steps, we deduce (see lemma 5 in [4]) that, in $B_{1 / 8}^{\prime}\left(\frac{1}{4} e_{n}\right) \times\left(-C \frac{\delta}{\mu}, C \frac{\delta}{\mu}\right), z$ is monotone increasing along the directions $\tau$ belonging to a cone $\Gamma^{x}\left(v^{*}, \theta^{*}\right)$ with

$$
\frac{\pi}{2}-\theta^{*} \leq b_{0}\left(\frac{\pi}{2}-\theta\right)
$$

Also, by deleting a fixed small neighborhood of a suitable generatrix of $\Gamma^{x}\left(v^{*}, \theta^{*}\right)$, we can write, for the remaining directions $\tau$,

$$
D_{\tau} z \geq c \delta D_{n} z
$$

Using (4.7) we infer that if $\epsilon_{0}^{\bar{a}} \ll \delta$,

$$
D_{\tau} u=D_{\tau} u-D_{\tau} z+D_{\tau} z \geq\left(c \delta-C \epsilon_{0}^{\bar{a}}\right) D_{n} z \geq \tilde{c} \delta D_{n} z
$$

and the conclusion follows easily.

The enlargement of the cone in time is more delicate. To say that in some region $u$ is monotone along the directions of a cone $\Gamma^{t}\left(e_{n}, \theta_{t}\right)$ means (see (ii) above) that there exist real numbers $A$ and $B$ such that

$$
0 \leq B-A \leq c \mu \quad\left(\mu=\frac{\pi}{2}-\theta\right)
$$


and

$$
A \leq-\frac{D_{t} u^{+}}{D_{n} u^{+}} \leq B \quad \text { or } \quad A \leq-\frac{D_{t} u^{-}}{D_{n} u^{-}} \leq B .
$$

We have the following result:

LEMMA 5.2 (Interior Gain in Time). Let $u$ be our solution of the f.b.p. and $\epsilon_{0}^{\bar{a}} \ll$ $\delta \ll \mu^{3}$. There exists a constant $\widetilde{\kappa}$ such that in

$$
B_{1 / 16}^{\prime}\left(\frac{1}{8} e_{n}\right) \times\left(-C \frac{\delta}{\mu}, C \frac{\delta}{\mu}\right)
$$

either

$$
-\frac{D_{t} u^{+}}{D_{n} u^{+}} \leq B-\widetilde{\kappa} \mu \quad \text { or } \quad-\frac{D_{t} u^{-}}{D_{n} u^{-}} \geq A+\widetilde{\kappa} \mu .
$$

Proof. Since $z$ is monotone along the directions of the cone $\Gamma^{t}\left(e_{n}, \hat{\theta}^{t}\right)$, according to the choice of $\hat{\theta}^{t}$, there is a constant $c_{0}^{*}$ such that

$$
0 \leq B-A \leq c_{0}^{*} \mu_{0} \quad\left(\mu_{0}=\frac{\pi}{2}-\hat{\theta}_{0}^{t}\right)
$$

and

all over inside $\mathcal{C}_{1}^{*}$.

$$
A \leq-\frac{D_{t} z^{+}}{D_{n} z^{+}} \leq B \quad \text { or } \quad A \leq-\frac{D_{t} z^{-}}{D_{n} z^{-}} \leq B
$$

After two iterations of lemma 8 in [4] we have, in $B_{1 / 16}^{\prime}\left(\frac{1}{8} e_{n}\right) \times\left(-C \frac{\delta}{\mu}, C \frac{\delta}{\mu}\right)$ : either

$$
-\frac{D_{t} z^{+}}{D_{n} z^{+}} \leq B-\widetilde{\kappa}^{*} \mu \quad \text { or } \quad-\frac{D_{t} z^{-}}{D_{n} z^{-}} \geq A+\widetilde{\kappa}^{*} \mu,
$$

respectively, when

$$
G\left(\alpha_{+}^{*}, \alpha_{-}^{*}\right) \geq-\frac{A+B}{2} \text { or } \quad G\left(\alpha_{+}^{*}, \alpha_{-}^{*}\right) \leq-\frac{A+B}{2},
$$

where $\alpha_{ \pm}^{*}=\left|\nabla z\left( \pm \frac{1}{8} e_{n}, 0\right)\right|$.

Assume the first one of the two above inequalities holds (if the other one holds, the argument is the same). Then

$$
D_{t} z^{+}+B D_{n} z^{+}-\widetilde{\kappa}^{*} \mu D_{n} z^{+} \geq 0 .
$$

Thus, from 4.7) and 4.8 we obtain

$$
D_{t} u+C \epsilon_{0}^{\bar{a}} D_{n} u(p)+\left(B-\widetilde{\kappa}^{*} \mu\right)\left(1+C \epsilon_{0}^{\bar{a}}\right) D_{n} u \geq 0
$$

that is,

$$
\begin{aligned}
-\frac{D_{t} u^{+}}{D_{n} u^{+}} & \leq\left(B-\widetilde{\kappa}^{*} \mu\right)\left(1+C \epsilon_{0}^{\bar{a}}\right)+C \epsilon_{0}^{\bar{a}} \\
& \leq B-\widetilde{\kappa}^{*} \mu+C \epsilon_{0}^{\bar{a}}\left(1+B-\widetilde{\kappa}^{*} \mu\right) \\
& \leq B-\widetilde{\kappa} \mu
\end{aligned}
$$


since $\epsilon_{0}^{\bar{a}} \ll \mu^{3}$ and $|B| \leq \tan \mu$.

\section{Basic Iteration Steps and Proof of Theorem 1.2}

We want now to propagate the gain in the opening of the space and space-time cones of monotonocity to the free boundary in a smaller cylinder. To apply lemma 12 in [4], we need a refinement of the Hopf principle in lemma 5.1 of [5] due to the lack of control of $D_{t} u$ by $u_{x_{n}}$ up to the free boundary. We are dealing with the following situation, which occurs along the double-iteration process mentioned in the introduction.

Let

$$
\mathscr{Q}:=\left\{\left(x^{\prime}, x_{n}, t\right):\left|x^{\prime}\right|<2, f\left(x^{\prime}, t\right)<x_{n}<2,-2<t<2\right\},
$$

and define $\Omega_{0}:=\mathscr{Q} \cap\{t=0\}$,

$$
F:=\left\{\left(x^{\prime}, x_{n}, t\right):\left|x^{\prime}\right|<2, x_{n}=f\left(x^{\prime}, t\right),-2<t<2\right\} \cap\{t=0\}
$$

and $F_{0}:=F \cap\{t=0\}$.

Let $w$ be a solution of $w_{t}-\mathscr{L} w=0$ in $\mathscr{Q}$, positive and continuous in $\overline{\mathscr{Q}}$, vanishing on $F$.

Assume that there exists a sequence of spatial cones $\Gamma_{k}=\Gamma^{x}\left(\theta_{k}, e_{k}\right)$, real numbers $C_{0}, \bar{c}$, and $\rho$, integers $k_{0}$ and $\bar{k}, \bar{k} \gg 1$, and a decreasing sequence $\left\{\delta_{k}\right\}$ with the following properties:

(a) $\delta_{k} \leq C_{0} /(k+\bar{k})^{1+\rho},\left|e_{k}-e_{k+1}\right| \leq c \delta_{k}$;

(b) for $k \leq k_{0}$,

$$
\Gamma_{k} \cap\left(B_{4^{-k}(0)}^{\prime} \backslash B_{4^{-k-1}}^{\prime}(0)\right) \subset \Omega_{0} \cap\left(B_{4^{-k}(0)}^{\prime} \backslash B_{4^{-k-1}}^{\prime}(0)\right) ;
$$

(c) there exists a ball $B_{4^{-k_{0}}}^{\prime} \subset \Omega_{0}$ tangent to $F_{0}$ at 0 , with inward normal $e_{k_{0}}$. Then we can prove the following Hopf principle:

LEMMA 6.1. Under the above conditions, there exists a positive constant $C^{*}=$ $C^{*}\left(n, C_{0}, \bar{c}, \rho\right)$ such that, near $(0,0)$,

$$
w(x, 0) \geq C^{*} w_{m}\left\langle x, e_{k_{0}}\right\rangle
$$

where $w_{m}=\min \left\{w(x, t): x_{n}=2\right\}$.

PROOF. The proof follows closely the proof of lemma 5.1 in [5], by first constructing a Lyapunov-Dini domain

$$
\Omega^{\prime}=\left\{\left(x^{\prime}, x_{n}\right):\left|x^{\prime}\right|<2, g\left(x^{\prime}\right)<x_{n}<2\right\}
$$

such that

(i) $g\left(x^{\prime}\right)>f\left(x^{\prime}, 0\right)$ for every $x^{\prime},\left|x^{\prime}\right|<2$ and $g(0) \sim 4^{-k_{0}}$, and

(ii) $g \in C^{1}$ and $\omega_{\nabla g}(s) \leq C_{1}|\log (2 / s)|^{1-\rho / 2}$ for $s$ small, where $\omega_{\nabla g}$ denotes the modulus of continuity of $\nabla g$. 
Moreover, due to the flatness in space of $F(u)$, we can easily construct a function $G=G\left(x^{\prime}, t\right)$, Lipschitz in space and time, flat in space, such that $G\left(x^{\prime}, t\right)>$ $f\left(x^{\prime}, 0\right)$ for every $x^{\prime},\left|x^{\prime}\right|<2,|t|<2$, and $G\left(x^{\prime}, 0\right)=g\left(x^{\prime}\right)$. Define

$$
\mathscr{Q}^{\prime}=\left\{\left(x^{\prime}, x_{n}, t\right):\left|x^{\prime}\right|<2, G\left(x^{\prime}, t\right)<x_{n}<2,-2<t<2\right\},
$$

and let $v$ be the solution of $D_{t} v-\mathscr{L} v=0$ in $\mathscr{Q}^{\prime}$, with $v=w_{m}$ on $x_{n}=2$ and $v$ vanishing everywhere else on $\partial_{p} \mathscr{Q}^{\prime}$. By the maximum principle, $w>v$ in $\mathscr{Q}^{\prime}$. On the other hand, by Lemma 2.2 (a), we infer that, near the origin,

$$
\left|D_{t} v(x, 0)\right| \leq c d_{x, 0}^{a-1} D_{n} v(x, 0) .
$$

Thus, at the level $t=0, v$ is a supersolution of an elliptic equation satisfying the hypotheses of [21, theorem 3.1]. Thus we can write

$$
4^{k_{0}} w\left(e_{k_{0}}\right) \geq 4^{k_{0}} v\left(e_{k_{0}}\right) \geq C^{*} w_{m},
$$

and therefore, by condition (c), 6.1) is true for $w$.

Now we have all the ingredients to reproduce the proof of lemma 12 in [4]. Due again to the lack of the a priori nondegeneracy assumed in that paper and moreover to the restriction

$$
\omega \equiv \omega(1) \leq \epsilon_{0}^{(1-\alpha) / \alpha},
$$

it is possible to carry the interior gains only for $\epsilon_{0}$-monotonicity. The enlargement of the cones allows us to obtain an improvement in the $\epsilon_{0}$-monotonicity, passing from $\epsilon_{0}$ to $\epsilon_{1}<\epsilon_{0}$, still keeping the validity of (6.2) thanks to the improvement of the modulus of continuity of the coefficients from $\omega$ to $\omega_{1}<\omega$. These are the first and basic steps of the combined iteration procedure mentioned in the introduction. Specifically, the following lemma holds:

LEMMA 6.2. Let $u$ be a solution of our f.b.p. in $\mathscr{C}_{2,2}$. Assume that $\delta=\delta\left(n, L_{0}\right.$, $\lambda, \Lambda)$ is small enough and $\epsilon_{0} \ll \delta \ll \mu^{3}$. Let (6.2) hold, $\|A-I\|_{\infty} \leq \omega$, and $\|\mathbf{b}\|_{\infty} \leq \omega$. There exist positive constants $c_{1}, c_{2}, c$, and $C$ and unit vectors $e$ (spatial), $v_{1} \in \operatorname{span}\left\{e, e_{t}\right\}$ such that, in

$$
B_{4^{-2}}^{\prime} \times\left(-C \frac{\delta}{4 \mu}, C \frac{\delta}{4 \mu}\right),
$$

$u$ is $\epsilon_{0}$-monotone in $\Gamma^{x}\left(e, \theta_{1}\right) \cup \Gamma^{t}\left(v_{1}, \theta_{1}^{t}\right)$ with

$$
\begin{aligned}
& \frac{\pi}{2}-\theta_{1} \equiv \delta_{1} \leq \delta-c_{1} \frac{\delta^{2}}{\mu}, \\
& \frac{\pi}{2}-\theta_{1}^{t} \equiv \mu_{1} \leq \mu-c_{2} \delta,
\end{aligned}
$$

and $\delta_{1} \ll \mu_{1}^{3}$, $\left|e-e_{n}\right| \leq c \delta$, and $\left|\nu_{1}-v\right| \leq c \mu$. 
PROOF. Let $\phi_{\eta}$ be the family constructed in Lemma 3.3, and in

$$
D^{+}=\left(B_{1}^{\prime} \backslash B_{1 / 8}^{\prime}\left(\frac{5}{8} e_{n}\right)\right) \times(-1,1)
$$

set

$$
\bar{V}_{\eta}(p) \equiv \sup _{q \in B_{\epsilon_{0} \phi_{\eta}}(p)} u(q-\tau)+c \epsilon \delta u\left(p_{0}^{+}\right) w_{\mathscr{L}}^{+}(p),
$$

where $\tau$ is a small vector belonging to $\Gamma^{x}\left(e_{n}, \theta-\delta\right) \cup \Gamma^{t}\left(e_{n}, \theta_{t}-\mu\right)$, with $\epsilon_{0}=$ $|\tau| \sin \delta$, and let $w_{\mathscr{L}}^{+}$be the $\mathscr{L}$-caloric measure in $\Omega^{+}\left(\bar{v}_{0}\right)$ of $\Sigma^{+}=\partial B_{1 / 8}^{\prime}\left(\frac{5}{8} e_{n}\right) \times$ $(-1,1) . \quad \bar{V}_{\eta}(p)$ is a $\mathscr{L}$-subsolution in the complement of its zero set. At this point it is possible to reproduce exactly the proof of lemma 14 in [4] to reach the conclusions, using the crucial Lemma 6.1 .

For the improvement of the $\epsilon_{0}$-monotonicity we use the results in [11, secs. 2-4] that actually apply to a more general case. In particular, the first part of the proof of theorem 4.1 in [11] gives the following lemma:

Lemma 6.3. Assume the hypotheses of Lemma 6.2 hold. Then there exist positive constants $\lambda<1$, close to $1, \alpha^{\prime}<\beta^{\prime}<\frac{1}{2}$, and $\widetilde{c}$ such that $u$ is $\lambda \epsilon_{0}$-monotone along the directions of

$$
\Gamma^{x}\left(e, \theta_{1}-\tilde{c} \epsilon_{0}^{\beta^{\prime}}\right) \cup \Gamma^{t}\left(v_{1}, \theta_{1}^{t}-\tilde{c} \epsilon_{0}^{\beta^{\prime}}\right)
$$

in

$$
B_{4^{-2}\left(1-\tilde{c} \epsilon_{0}^{\alpha^{\prime}}\right)}^{\prime} \times\left(-C \frac{\delta}{4 \mu}+\tilde{c} \epsilon_{0}^{\alpha^{\prime}}, C \frac{\delta}{4 \mu}-\tilde{c} \epsilon_{0}^{\alpha^{\prime}}\right)
$$

We are now ready to end the proof of our main theorem.

Proof OF THEOREM 1.2. We shall apply inductively Lemmas 6.2 and 6.3 as in section 9.9 of [5] using the rescaling

$$
u_{k}(x, t)=5^{k} u\left(\frac{1}{5^{k}} x, \frac{\delta_{k}}{5^{k} \mu_{k}} t\right)
$$

where $\delta_{k}$ and $\mu_{k}$ verify the recurrence relations

$$
\begin{aligned}
\delta_{k+1} & \leq \delta_{k}-c_{1} \frac{\delta_{k}}{\mu_{k}}, \\
\mu_{k+1} & \leq \mu_{k}-c_{2} \delta_{k},
\end{aligned}
$$

and $\delta_{k} \ll \mu_{k}{ }^{3}$. The only difference is that we have to take care of the iteration of the restriction condition 6.2 that reads

$$
\omega_{k} \leq \bar{\epsilon}_{k}^{(1-\alpha) / \alpha}
$$

where $\omega_{k}$ and $\bar{\epsilon}_{k}$ come from the rescaling of the modulus of continuity of the coefficients of the operator $\mathscr{L}$ and of the $\epsilon_{k}$-monotonicity at each step. More precisely, if $\alpha=1$, that is, if the coefficients are Lipschitz-continuous, we may 
repeat verbatim the same arguments in [5] and use the same sequences $\delta_{k}, \mu_{k}$, and $\epsilon_{k}$ given by, for example,

$$
\begin{gathered}
\delta_{k} \sim \frac{1}{(k+\bar{k})^{4 / 3}}, \quad \bar{k} \gg 1, \\
\mu_{k} \sim \frac{1}{k^{1 / 3}}, \quad \epsilon_{k}=\frac{1}{30^{k+\bar{k}}} \quad(k \geq 1, \bar{k} \text { large }) .
\end{gathered}
$$

We obtain that in the sequence of cylinders

$$
B_{5^{-k}}^{\prime} \times\left(-C \frac{\delta_{k}}{5^{k} \mu_{k}}, C \frac{\delta_{k}}{5^{k} \mu_{k}}\right)
$$

$u$ is $\epsilon_{k+1}$-monotone along the directions $\tau$ belonging to

$$
\Gamma^{x}\left(\theta_{k}, e_{k}\right) \cup \Gamma^{x}\left(\theta_{k}^{t}, v_{k}\right)
$$

where $v_{k} \in \operatorname{span}\left\{e_{k}, e_{t}\right\}$. Notice that in this case 6.7) is automatically satisfied since $\omega_{k}$ is of order $\omega_{0} 5^{-k} \delta_{k} / \mu_{k}$.

We conclude that at each time level $t_{0},-1<t_{0}<1, \Omega^{ \pm}(u) \cap\left\{t=t_{0}\right\}$ is a Lyapunov-Dini domain and the conclusion follows as in [5].

If $0<\alpha<1, \omega_{k}$ is of order $\omega_{0} 5^{-\alpha k}\left(\delta_{k} / \mu_{k}\right)^{\alpha}$ and $\bar{\epsilon}_{k}$ of order $5^{k}\left(\mu_{k} / \delta_{k}\right) \epsilon_{k}$ so that, to repeat the arguments in [5, sec. 9], we choose, e.g., $\epsilon_{k}=\epsilon_{0} 5^{-2 k /(1-\alpha)}$.

This completes the proof of the theorem.

\section{Appendix: Proof of Theorem 3.1}

We show that $v_{\phi, \tau}$ is a viscosity subsolution for the operator $\mathscr{L}_{A, \mathbf{b}^{\prime}}-D_{t}$ in $\Omega^{+}\left(v_{\phi, \tau}\right)$; the other cases are analogous. To prove it, we show that for every $p \in \Omega^{+}\left(v_{\phi, \tau}\right)$

$$
\begin{aligned}
& \int_{|y|=1} v_{\phi, \tau}\left(x+r A^{1 / 2}(p) \sigma, t\right) d \mathscr{H}^{n-1}(\sigma) \\
& \geq v_{\phi, \tau}(p)+c_{n}\left(-\liminf _{s \rightarrow 0^{+}} \frac{v_{\phi, \tau}\left(p+s \mathbf{b}^{\prime}(p)\right)-v_{\phi, \tau}(p)}{s}\right. \\
& \left.\quad+\limsup _{s \rightarrow 0^{+}} \frac{v_{\phi, \tau}(x, t+s)-v_{\phi, \tau}(p)}{s}\right) r^{2}+o\left(r^{2}\right),
\end{aligned}
$$

as $r \rightarrow 0$.

Let $u$ be a solution of

$$
D_{t} u(p)=\operatorname{Tr}\left(B(p) D^{2} u(p)\right)+\mathbf{b}(p) \cdot \nabla u(p)
$$

in $\Omega \subset \mathbb{R}^{n+1}$, where as usual $p=(x, t) \in \mathbb{R}^{n+1}$ and $B(p) \in \mathscr{S}_{\lambda, \Lambda}(\Omega) \subset$ $\mathbb{S}^{n \times n}$. For every $t$, the function $u(\cdot, t)$ satisfies in $\Omega_{t}=\left\{x \in \mathbb{R}^{n}: p \in \Omega\right\}$ the nonhomogeneous elliptic equation

$$
\operatorname{Tr}\left(B(\cdot, t) D^{2} u(\cdot, t)\right)+\mathbf{b}(\cdot, t) \cdot \nabla u(\cdot, t)=D_{t} u(\cdot, t) .
$$


For simplicity we write $h_{\sigma, 0}(p)$ instead of $\left(A(p)^{1 / 2} \sigma, 0\right)$.

Let now $b$ be such that $|b| \leq \phi(p)$. Then

$$
v_{\phi}(p) \geq u\left(V_{r}(p), t+b\right),
$$

where $\left(\varsigma \in \mathbb{R}^{n}\right)$

since

$$
V_{r}(p)=x+\sqrt{\phi(p)^{2}-b^{2}} \frac{v_{s}^{*}+r \varsigma}{\left|v_{s}^{*}+r \varsigma\right|},
$$

$$
\left(V_{r}(p), t+b\right) \in \partial B_{\phi(p)}(p) .
$$

Similarly, if $b$ is such that $|b| \leq \phi\left(p+r h_{\sigma, 0}(p)\right)$, where $A \in \mathscr{S}_{\lambda, \Lambda}$, we get

$$
\begin{aligned}
v_{\phi}\left(p+r h_{\sigma, 0}(p)\right) & =\sup _{|\xi|=1} u\left(p+r h_{\sigma, 0}(p)+\phi\left(p+r h_{\sigma, 0}(p)\right) \xi\right) \\
& \geq u\left(V_{r}\left(p+r h_{\sigma, 0}(p)\right), t+b\right) .
\end{aligned}
$$

If $v \in \mathbb{R}^{n}$ is a unit vector, $\varsigma \in \mathbb{R}^{n}$, we have as $r \rightarrow 0$ :

$$
\frac{1}{|v+r \varsigma|}=1-r\langle v, \varsigma\rangle-\frac{r^{2}}{2}|\varsigma|^{2}+\frac{3}{2} r^{2}\langle v, \varsigma\rangle^{2}+o\left(r^{2}\right) \text {. }
$$

Thus

$$
\begin{aligned}
\frac{v+r \varsigma}{|v+r \varsigma|}= & v+r(-\langle v, \varsigma\rangle v+\varsigma) \\
& +r^{2}\left(-\langle v, \varsigma\rangle \varsigma-\frac{|v|^{2}}{2} v+\frac{3}{2}\langle v, \varsigma\rangle^{2} v\right)+o\left(r^{2}\right)
\end{aligned}
$$

and

$$
\begin{aligned}
\phi\left(p+r h_{\sigma, 0}(p)\right)= & \phi(p)+\left\langle\nabla \phi(p), A^{1 / 2}(p) \sigma\right\rangle r \\
& +\frac{1}{2}\left\langle D^{2} \phi(p) A^{1 / 2}(p) \sigma, A^{1 / 2}(p) \sigma\right\rangle r^{2}+o\left(r^{2}\right) .
\end{aligned}
$$

Hence if $P_{\tau}=p-\tau+\phi(p) v$, where $v=v_{s}^{*}+\kappa e_{t}$, then

$$
\begin{aligned}
& v_{\phi, \tau}\left(p+r h_{\sigma, 0}(p)\right) \geq u\left(\left(V\left(p+r h_{\sigma, 0}(p)\right), t+b\right)-\tau\right) \\
& \quad=u\left(\left(x+r A^{1 / 2}(p) \sigma+\sqrt{\phi\left(p+r h_{\sigma, 0}(p)\right)^{2}-b^{2}} \frac{v_{s}^{*}+r \varsigma}{\left|v_{s}^{*}+r \varsigma\right|}, t+b\right)-\tau\right) \\
& =u\left(P_{\tau}\right)+r\left\langle\nabla u\left(P_{\tau}\right), \mathscr{A}\right\rangle+r^{2}\left(\left\langle\nabla u\left(P_{\tau}\right), \mathscr{B}\right\rangle+\frac{1}{2}\left\langle D^{2} u\left(P_{\tau}\right) \mathscr{A}, \mathscr{A}\right\rangle\right)+o\left(r^{2}\right),
\end{aligned}
$$

where

$$
\mathscr{A}=\left\langle\nabla \phi(p), A^{1 / 2}(p) \sigma\right\rangle v+\phi(p)\left(\varsigma-\langle\nu, \varsigma\rangle v+A^{1 / 2}(p) \sigma\right)
$$

and

$$
\begin{aligned}
\mathscr{B}= & \frac{1}{2}\left\langle D^{2} \phi(p) A^{1 / 2}(p) \sigma, A^{1 / 2}(p) \sigma\right\rangle v+\left\langle\nabla \phi(p), A^{1 / 2}(p) \sigma\right\rangle(-\langle v, \varsigma\rangle v+\varsigma) \\
& -\phi(p)\left(\langle v, \varsigma\rangle \varsigma+\frac{|\varsigma|^{2}}{2} v-\frac{3}{2}\langle v, \varsigma\rangle^{2} v\right) .
\end{aligned}
$$


Integrating over $|\sigma|=1$ we get

$$
f_{|\sigma|=1}\left\langle\nabla u\left(P_{\tau}\right), \mathscr{A}\right\rangle d \mathscr{H}^{n-1}(\sigma)=0
$$

and

$$
\begin{aligned}
\text { (A.4) } f_{|\sigma|=1}\left\langle\nabla u\left(P_{\tau}\right), \mathscr{B}\right\rangle d \mathscr{H}^{n-1}(\sigma)= \\
\quad \frac{1}{2 n}\left\{\operatorname{Tr}\left(A(p) D^{2} \phi(p)\right)-\phi(p)\left[|\varsigma|^{2}-\langle\nu, \varsigma\rangle^{2}\right]\right\}\left|\nabla u\left(P_{\tau}\right)\right|+o\left(r^{2}\right) .
\end{aligned}
$$

Similarly,

$$
\begin{aligned}
\left\langle D^{2} u\right. & \left.\left(P_{\tau}\right) \mathscr{A}, \mathscr{A}\right\rangle \\
= & \left\langle D^{2} u\left(P_{\tau}\right) v, v\right\rangle\left\langle\nabla \phi(p), A^{1 / 2}(p) \sigma\right\rangle^{2} \\
& +\phi^{2}(p)\left\langle D^{2} u\left(P_{\tau}\right)(\varsigma-\langle v, \varsigma\rangle v),(\varsigma-\langle v, \varsigma\rangle v)\right\rangle \\
& +\left\langle D^{2} u\left(P_{\tau}\right) A^{1 / 2}(p) \sigma, A^{1 / 2}(p) \sigma\right\rangle \\
& +2 \phi(p)\left\langle D^{2} u\left(P_{\tau}\right) v,(\varsigma-\langle v, \varsigma\rangle v)\right\rangle\left\langle\nabla \phi(p), A^{1 / 2}(p) \sigma\right\rangle \\
& +2\left\langle D^{2} u\left(P_{\tau}\right) v, A^{1 / 2}(p) \sigma\right\rangle+2\left\langle D^{2} u\left(P_{\tau}\right)(\varsigma-\langle v, \varsigma\rangle v), A^{1 / 2}(p) \sigma\right\rangle .
\end{aligned}
$$

Thus

$$
\begin{aligned}
f_{|\sigma|=1} & \left\langle D^{2} u\left(P_{\tau}\right) \mathscr{A}, \mathscr{A}\right\rangle d \mathscr{H}^{n-1}(\sigma) \\
= & \frac{\left\langle D^{2} u\left(P_{\tau}\right) v, v\right\rangle\left|A^{1 / 2}(p) \nabla \phi(p)\right|^{2}}{n} \\
& +\frac{\phi^{2}(p)}{n}\left\langle D^{2} u\left(P_{\tau}\right)(\varsigma-\langle v, \varsigma\rangle v),(\varsigma-\langle v, \varsigma\rangle v)\right\rangle \\
& +\frac{1}{n} \operatorname{Tr}\left(A(p) D^{2} u\left(P_{\tau}\right)\right)+o\left(r^{2}\right)=\frac{1}{n} \operatorname{Tr}\left(F(p) D^{2} u\left(P_{\tau}\right)\right)+o\left(r^{2}\right),
\end{aligned}
$$

where

$$
\begin{aligned}
F(p)= & v \otimes v\left|A(p)^{1 / 2} \nabla \phi(p)\right|^{2} \\
& +\phi^{2}(p)(\varsigma-\langle v, \varsigma\rangle v) \otimes(\varsigma-\langle v, \varsigma\rangle v)+A(p) .
\end{aligned}
$$

Set $\zeta_{n}=\left\langle F(p) v_{n}, v_{n}\right\rangle / n \beta_{n}$ and $\mathscr{T}_{p}(\phi)=\operatorname{Tr}\left(A(p) D^{2} \phi(p)\right)$. Choosing $\varsigma$ as in Lemma A.3 below, we may reproduce the matrix $B$ and write

$$
\operatorname{Tr}\left(F(p) D^{2} u\left(P_{\tau}\right)\right) \geq \frac{\left\langle F(p) v_{n}, v_{n}\right\rangle}{\beta_{n}} \operatorname{Tr}\left(B\left(P_{\tau}\right) D^{2} u\left(P_{\tau}\right)\right) .
$$


As a consequence,

$$
\begin{aligned}
& \int_{|\sigma|=1} v_{\phi, \tau}\left(p+r h_{\sigma, 0}(p)\right) d \mathscr{H}^{n-1}(\sigma) \\
& \geq v_{\phi, \tau}(p)+\left(\frac{1}{n} \operatorname{Tr}\left(F D^{2} u\left(P_{\tau}\right)\right)\right. \\
& \left.\quad+\frac{1}{2 n}\left\{\mathscr{T}_{p}(u)-\phi(p)\left[|\zeta|^{2}-\langle v, \varsigma\rangle^{2}\right]\right\}\left|\nabla u\left(P_{\tau}\right)\right|\right) r^{2}+o\left(r^{2}\right) \\
& \geq v_{\phi, \tau}(p)+\zeta_{n} \operatorname{Tr}\left(B\left(P_{\tau}\right) D^{2} u\left(P_{\tau}\right)\right) \\
& \quad+\frac{1}{2 n}\left\{\mathscr{T}_{p}(u)-\phi(p)\left[|\zeta|^{2}-\langle v, \zeta\rangle^{2}\right]\right\}\left|\nabla u\left(P_{\tau}\right)\right| r^{2}+o\left(r^{2}\right) \\
& =v_{\phi, \tau}(p)+\zeta_{n}\left(D_{t} u\left(P_{\tau}\right)-\mathbf{b}\left(P_{\tau}\right) \cdot \nabla u\left(P_{\tau}\right)\right) \\
& \quad+\frac{1}{2 n}\left\{\mathscr{T}_{p}(u)-\phi(p)\left[|\varsigma|^{2}-\langle v, \varsigma\rangle^{2}\right]\right\}\left|\nabla u\left(P_{\tau}\right)\right| r^{2}+o\left(r^{2}\right) .
\end{aligned}
$$

Since $D \phi_{t}>0$, we get

$$
\begin{aligned}
& v_{\phi, \tau}\left(p+h e_{t}\right)-v_{\phi}(p) \\
& =u\left(\left(p+h e_{t}\right)+\tau+\phi\left(p+h e_{t}\right) v_{\left(p+h e_{t}\right)}\right) \\
& \quad-u\left(p+\tau+\phi(p) v_{(p)}\right) \\
& =\left\langle\nabla u\left(P_{\tau}\right),\left(\phi\left(p+h e_{t}\right)-\phi(p)\right) v_{\left(p+h e_{t}\right)}^{s}+\phi(p)\left(v_{\left(p+h e_{t}\right)}^{s}-v_{(p)}^{s}\right)\right\rangle \\
& \quad+D_{t} u\left(P_{\tau}\right)\left(\left(\phi\left(p+h e_{t}\right)-\phi(p)\right) v_{\left(p+h e_{t}\right)}^{t}\right. \\
& \left.\quad+\phi(p)\left(v_{\left(p+h e_{t}\right)}^{t}-v_{(p)}^{t}\right)+h\right)+o(h) .
\end{aligned}
$$

Hence

$$
\begin{aligned}
& -\limsup _{h \rightarrow 0^{+}} \frac{v_{\phi, \tau}\left(p+h e_{t}\right)-v_{\phi, \tau}(p)}{h} \\
& \quad \geq-\left\langle\nabla u\left(P_{\tau}\right), D_{t} \phi(p) v_{(p)}^{s}\right\rangle-D_{t} u\left(P_{\tau}\right)\left(D_{t} \phi(p)\left|v_{(p)}^{t}\right|+1\right) \\
& \quad=-\left|\nabla u\left(P_{\tau}\right)\right| D_{t} \phi(p)-D_{t} u\left(P_{\tau}\right)\left(D_{t} \phi(p)\left|v_{(p)}^{t}\right|+1\right) .
\end{aligned}
$$

Observe now that

$$
\begin{aligned}
v_{\phi\left(p+s \mathbf{b}^{\prime}(p)\right), \tau}\left(p+s \mathbf{b}^{\prime}(p)\right)-v_{\phi(p), \tau}(p) \\
\geq u\left(p+s \mathbf{b}^{\prime}(p)+\tau+v^{*} \phi\left(p+s \mathbf{b}^{\prime}(p)\right)\right)-u\left(p+\tau+v^{*} \phi(p)\right) \\
=u\left(p+s \mathbf{b}^{\prime}(p)+\tau+v^{*} \phi(p)+s v^{*} \phi(p)\left\langle\mathbf{b}^{\prime}(p), \nabla \phi(p)\right\rangle+o(s)\right) \\
\quad-u\left(p+s \mathbf{b}^{\prime}\left(P_{\tau}\right)+\tau+v^{*}\left(\phi(p)+s\left\langle\mathbf{b}^{\prime}\left(P_{\tau}\right), \nabla \phi(p)\right\rangle+o(s)\right)\right) \\
\quad+u\left(p+s \mathbf{b}^{\prime}\left(P_{\tau}\right)+\tau+v^{*}\left(\phi(p)+s\left\langle\mathbf{b}^{\prime}\left(P_{\tau}\right)\right), \nabla \phi(p)\right\rangle+o(s)\right) \\
\quad-u\left(p+\tau+v^{*} \phi(p)\right) .
\end{aligned}
$$


(recalling that $P_{\tau}=p+\tau+v^{*} \phi(p)$ )

$$
\begin{aligned}
= & u\left(P_{\tau}+s\left(\mathbf{b}^{\prime}(p)+v^{*} \phi(p)\left\langle\mathbf{b}^{\prime}(p), \nabla \phi(p)\right\rangle+o(1)\right)\right) \\
& -u\left(P_{\tau}+s\left(\mathbf{b}^{\prime}\left(P_{\tau}\right)+v^{*} \phi(p)\left\langle\mathbf{b}^{\prime}(p), \nabla \phi\left(P_{\tau}\right)\right\rangle+o(1)\right)\right) \\
& +u\left(P_{\tau}+s\left(\mathbf{b}^{\prime}\left(P_{\tau}\right)+v^{*} \phi(p)\left\langle\mathbf{b}^{\prime}(p), \nabla \phi\left(P_{\tau}\right)\right\rangle+o(1)\right)\right)-u\left(P_{\tau}\right),
\end{aligned}
$$

(by Taylor's formula)

$$
\begin{aligned}
= & s\left\langle\nabla u\left(P_{\tau}\right), v^{*} \phi(p)\left\langle\left(\mathbf{b}^{\prime}(p)-\mathbf{b}^{\prime}\left(P_{\tau}\right)\right), \nabla \phi(p)\right\rangle\right\rangle \\
& +s\left\langle\nabla u\left(P_{\tau}\right),\left\langle\nabla \phi(p), \mathbf{b}^{\prime}\left(P_{\tau}\right)\right\rangle v^{*}+\mathbf{b}^{\prime}\left(P_{\tau}\right)\right\rangle+o(s),
\end{aligned}
$$

as $s \rightarrow 0$. As a consequence,

$$
\begin{aligned}
-\liminf _{s \rightarrow 0^{+}} \frac{v_{\phi\left(p+s \mathbf{b}^{\prime}(p)\right), \tau}\left(p+s \mathbf{b}^{\prime}(p)\right)-v_{\phi(p), \tau}(p)}{s} \\
\leq-\left\langle\nabla u\left(P_{\tau}\right),\left\langle\nabla \phi(p), \mathbf{b}^{\prime}\left(P_{\tau}\right)\right\rangle v^{*}+\mathbf{b}^{\prime}\left(P_{\tau}\right)\right\rangle \\
\quad-\left\langle\nabla u\left(P_{\tau}\right), v^{*} \phi(p)\left\langle\left(\mathbf{b}^{\prime}(p)-\mathbf{b}^{\prime}\left(P_{\tau}\right)\right), \nabla \phi(p)\right\rangle\right\rangle .
\end{aligned}
$$

Thus, since $D_{t} \phi \geq 0$, we get

$$
\begin{aligned}
r^{-2} f_{|\sigma|=1} & \left(v_{\phi, \tau}\left(p+r h_{\sigma, 0}(p)\right)-v_{\phi, \tau}(p)\right) d \mathscr{H}^{n-1}(\sigma) \\
& \quad-c_{n} \limsup _{h \rightarrow 0^{+}} \frac{v_{\phi}\left(p+h e_{t}\right)-v_{\phi}(p)}{h} \\
\geq & \zeta_{n}\left(D_{t} u\left(P_{\tau}\right)-\mathbf{b}\left(P_{\tau}\right) \cdot \nabla u\left(P_{\tau}\right)\right) \\
& +\frac{1}{2 n}\left\{\mathscr{T}_{p}(\phi)-\phi(p)\left[|\varsigma|^{2}-\langle v, \varsigma\rangle^{2}\right]\right\}\left|\nabla u\left(P_{\tau}\right)\right| \\
& -\left|\nabla u\left(P_{\tau}\right)\right| D_{t} \phi(p)-D_{t} u\left(P_{\tau}\right)\left(D_{t} \phi(p)\left|v_{p}^{t}\right|+1\right) \\
& \left.-\zeta_{n} \mathbf{b}\left(P_{\tau}\right) \cdot \nabla u\left(P_{\tau}\right)\right)+o(1) \\
= & \left(\zeta_{n}-1\right) D_{t} u\left(P_{\tau}\right)+\frac{1}{2 n}\left\{\mathscr{T}_{p}(\phi)-\phi(p)\left[|\varsigma|^{2}-\langle v, \varsigma\rangle^{2}\right]\right\}\left|\nabla u\left(P_{\tau}\right)\right| \\
& -\left|\nabla u\left(P_{\tau}\right)\right| D_{t} \phi(p)-D_{t} u\left(P_{\tau}\right) D_{t} \phi(p)\left|v_{(p)}^{t}\right|-\zeta_{n} \mathbf{b}\left(P_{\tau}\right) \cdot \nabla u\left(P_{\tau}\right)+o(1) .
\end{aligned}
$$

Since $\theta^{t} \geq \frac{\pi}{4}$, we have $\left|D_{t} u\left(P_{\tau}\right)\right| \leq c\left|\nabla u\left(P_{\tau}\right)\right|$, so that we can continue the above sequence of inequalities as follows:

$$
\begin{aligned}
\geq & -c\left|\zeta_{n}-1\right|\left|\nabla u\left(P_{\tau}\right)\right|+\frac{1}{2 n}\left\{\mathscr{T}_{p}(\phi)-\phi(p)\left[|\varsigma|^{2}-\langle v, \varsigma\rangle^{2}\right]\right\}\left|\nabla u\left(P_{\tau}\right)\right| \\
& -\left|\nabla u\left(P_{\tau}\right)\right| D_{t} \phi(p)-\left|\nabla u\left(P_{\tau}\right)\right| D_{t} \phi(p)\left|v_{(p)}^{t}\right|-\zeta_{n} \mathbf{b}\left(P_{\tau}\right) \cdot \nabla u\left(P_{\tau}\right)+o(1) .
\end{aligned}
$$


We now use A.6 and the estimates for $\varsigma$, given in A.15, Lemma A.3, to obtain

$$
\begin{aligned}
\geq & \left|\nabla u\left(P_{\tau}\right)\right|\left(-c|\nabla \phi(p)|+\frac{1}{2 n}\left\{\mathscr{T}_{p}(\phi)-C^{2} \frac{(|\nabla \phi(p)|+\omega)^{2}}{\phi(p)}\right\}-\left(1+\left|v_{p}^{t}\right|\right) D_{t} \phi(p)\right) \\
& +\left(-\zeta_{n}+c_{n}\right) \mathbf{b}\left(P_{\tau}\right) \cdot \nabla u\left(P_{\tau}\right)-c_{n} \mathbf{b}\left(P_{\tau}\right) \cdot \nabla u\left(P_{\tau}\right)+o(1) \\
= & \left|\nabla u\left(P_{\tau}\right)\right|\left(-c|\nabla \phi(p)|+\frac{1}{2 n}\left\{\mathscr{T}_{p}(\phi)-C^{2} \frac{(|\nabla \phi(p)|+\omega)^{2}}{\phi(p)}\right\}-\left(1+\left|v_{p}^{t}\right|\right) D_{t} \phi(p)\right) \\
& +\left(-\zeta_{n}+c_{n}\right)\left\langle\mathbf{b}\left(P_{\tau}\right), \nabla u\left(P_{\tau}\right)\right\rangle+c_{n}\left\langle\mathbf{b}^{\prime}\left(P_{\tau}\right), \nabla \phi(p)\right\rangle\left\langle\nabla u\left(P_{\tau}\right), v^{*}\right\rangle \\
& +c_{n}\left\langle\left(-\mathbf{b}\left(P_{\tau}\right)+\mathbf{b}^{\prime}\left(P_{\tau}\right)\right), \nabla u\left(P_{\tau}\right)\right\rangle \\
& +c_{n}\left\langle\nabla u\left(P_{\tau}\right), v^{*} \phi(p)\left\langle\left(\mathbf{b}^{\prime}(p)-\mathbf{b}^{\prime}\left(P_{\tau}\right)\right), \nabla \phi(p)\right\rangle\right\rangle \\
& -c_{n}\left(\left\langle\mathbf{b}^{\prime}\left(P_{\tau}\right), \nabla u\left(P_{\tau}\right)\right\rangle+\left\langle\mathbf{b}^{\prime}\left(P_{\tau}\right), \nabla \phi(p)\right\rangle\left\langle\nabla u\left(P_{\tau}\right), v^{*}\right\rangle\right) \\
& -c_{n}\left\langle\nabla u\left(P_{\tau}\right), v^{*} \phi(p)\left\langle\left(\mathbf{b}^{\prime}(p)-\mathbf{b}^{\prime}\left(P_{\tau}\right)\right), \nabla \phi(p)\right\rangle\right\rangle+o(1) .
\end{aligned}
$$

Taking into account A.7) and the boundedness of $\mathbf{b}, \mathbf{b}^{\prime}$, and $\phi$, we get

$$
\begin{array}{r}
\geq\left|\nabla u\left(P_{\tau}\right)\right|\left(-\widetilde{c}|\nabla \phi(p)|+\frac{1}{2 n}\left\{\mathscr{T}_{p}(\phi)-C^{2} \frac{(|\nabla \phi(p)|+\omega)^{2}}{\phi(p)}\right\}\right. \\
\left.-\left(1+\left|v_{p}^{t}\right|\right) D_{t} \phi(p)\right) \\
-c_{n} \liminf _{s \rightarrow 0^{+}} \frac{v_{\phi\left(p+s \mathbf{b}^{\prime}(p)\right), \tau}\left(p+s \mathbf{b}^{\prime}(p)\right)-v_{\phi(p), \tau}(p)}{s}+o(1) \\
\geq\left|\nabla u\left(P_{\tau}\right)\right| H\left(D^{2} \phi(p), \nabla \phi(p), D_{t} \phi(p)\right) \\
-\liminf _{s \rightarrow 0^{+}} \frac{v_{\phi\left(p+s \mathbf{b}^{\prime}(p)\right), \tau}\left(p+s \mathbf{b}^{\prime}(p)\right)-v_{\phi(p), \tau}(p)}{s}+o(1),
\end{array}
$$

where

$$
\begin{aligned}
H\left(D^{2} \phi, \nabla \phi, D_{t} \phi\right)= & \mathscr{T}_{p}(\phi)-C^{2} \frac{(|\nabla \phi(p)|+\omega)^{2}}{\phi(p)} \\
& -c_{2}(|\nabla \phi(p)|+\omega)-\left(1+\left|v_{p}^{t}\right|\right) D_{t} \phi(p) .
\end{aligned}
$$

In particular,

$$
\begin{aligned}
H\left(D^{2} \phi, \nabla \phi, D_{t} \phi\right) \geq & \mathscr{P}^{-}(\phi)-C^{2} \frac{(|\nabla \phi(p)|+\omega)^{2}}{\phi(p)} \\
& -c_{2}(|\nabla \phi(p)|+\omega)-\left(1+\left|v_{p}^{t}\right|\right) D_{t} \phi(p) \geq 0,
\end{aligned}
$$

so that (i) is proved. 
To prove (ii), we observe that

$$
w_{\phi, \tau}(p)=\inf _{B_{\phi(p)}(p)} u(q+\tau)=-\sup _{B_{\phi(p)}(p)}(-u(q+\tau))
$$

satisfies, in a viscosity sense, the inequality

$$
\operatorname{Tr}\left(A(p) D^{2} w_{\phi, \tau}(p)\right) \geq-\mathbf{b}^{\prime}(p) \cdot \nabla w_{\phi, \tau}(p)+D_{t} w_{\phi, \tau}(p) .
$$

Then $-w_{\phi, \tau}$ is still a subsolution of the same operator, and in particular, by linearity, $w_{\phi, \tau}$ satisfies (in a viscosity sense)

$$
\operatorname{Tr}\left(A(p) D^{2} w_{\phi, \tau}\right) \leq-\mathbf{b}^{\prime}(p) \cdot \nabla w_{\phi, \tau}+D_{t} w_{\phi, \tau} .
$$

Thus $w_{\phi, \tau}$ is a supersolution of the operator $\mathscr{L}_{A, \mathbf{b}^{\prime}}-D_{t}$.

\section{A.1 Choice of the Vector 5}

In this subsection we complete the proof of Lemma 3.1 by choosing the vector 5 (see Lemma A.3 below). For the reader's convenience, we first state two elementary lemmas from linear algebra, which we apply in the proof of Lemma A.3.

Lemma A.1. Let $B$ and $G$ be symmetric matrices. Assume that $B$ is positive definite. Let $\left\{v_{1}, v_{2}, \ldots, v_{n}\right\}$ be an orthonormal basis in $\mathbb{R}^{n}$ of $G$-eigenvectors corresponding to the eigenvalues $\left\{\lambda_{1} \leq \lambda_{2} \leq \cdots \leq \lambda_{n}\right\}$ of $G$. Similarly, let $\left\{w_{1}, w_{2}, \ldots, w_{n}\right\}$ be an orthonormal basis of $B$ eigenvectors, corresponding to the eigenvalues $\left\{0<\beta_{1} \leq \beta_{2} \leq \cdots \leq \beta_{n}\right\}$ of $B$. Then for every symmetric matrix $F$ :

(i) $\operatorname{Tr}(F G)=\sum_{i=1}^{n} \lambda_{i}\left\langle F v_{i}, v_{i}\right\rangle$, and

(ii) $\operatorname{Tr}(B G) \leq \sum_{i=1}^{n} \lambda_{i} \beta_{i}$

LemmA A.2. Let $\left\{v_{1}, v_{2}, \ldots, v_{n}\right\}$ be an orthogonal basis of $\mathbb{R}^{n}$. Then for every $k \neq j$ and $k \neq i$

$$
\begin{aligned}
{\left[v_{i} \otimes v_{i}-v_{j} \otimes v_{j}\right] v_{k} } & =0, \\
{\left[v_{i} \otimes v_{i}-v_{j} \otimes v_{j}\right] v_{i} } & =v_{i}, \\
{\left[v_{i} \otimes v_{i}-v_{j} \otimes v_{j}\right] v_{j} } & =-v_{j} .
\end{aligned}
$$

Moreover,

$$
\left[\begin{array}{c}
v_{1}^{T} \\
\vdots \\
v_{n}^{T}
\end{array}\right]\left[v_{i} \otimes v_{i}-v_{j} \otimes v_{j}\right]\left[v_{1}, v_{2}, \ldots, v_{n}\right]=e_{i} \otimes e_{i}-e_{j} \otimes e_{j} .
$$


Let now $\lambda_{i}(A)$ be the $i^{\text {th }}$ eigenvalue of the symmetric matrix $A$. We denote $\lambda_{\max }(A)=\max _{i=1, \ldots, n}\left\{\lambda_{i}(A)\right\}$ and $\lambda_{\min }(A)=\min _{i=1, \ldots, n}\left\{\lambda_{i}(A)\right\}$. Let $A, B \in$ $\mathscr{S}_{\lambda, \Lambda}$ be symmetric positive definite matrices and $0<\lambda \leq \Lambda$. Then

$$
\max \left\{\left|\lambda_{\max }(A-B)\right|,\left|\lambda_{\min }(A-B)\right|\right\} \leq \Lambda-\lambda
$$

and

$$
\max \left\{\left|\lambda_{\max }(A-B)\right|,\left|\lambda_{\min }(A-B)\right|\right\} \leq\|A-B\|_{\infty} .
$$

Finally we have the following result:

Lemma A.3. Assume the hypotheses of Theorem 3.1 are fulfilled. There exists a positive constant $C=C(\lambda, \Lambda, n)$ such that if $\|A-B\| \leq C(\Lambda / \lambda-1) \leq$ $\omega,\left\|\mathbf{b}-\mathbf{b}^{\prime}\right\|_{\infty} \leq \omega$, there exists a choice of $\varsigma \in \mathbb{R}^{n}$ such that if $F$ is the matrix defined in A.5, then

$$
\begin{gathered}
\frac{\left\langle F(p) v_{n}, v_{n}\right\rangle}{\beta_{n}} \operatorname{Tr}\left(B\left(P_{\tau}\right) D^{2} u\left(P_{\tau}\right)\right) \leq \operatorname{Tr}\left(F(p) D^{2} u\left(P_{\tau}\right)\right) \\
|\zeta|^{2} \leq C \frac{(|\nabla \phi(p)|+\omega)^{2}}{\phi(p)}
\end{gathered}
$$

and

(A.10) $\operatorname{Tr}\left(A(p) D^{2} \phi(p)\right)-\phi(p)\left[|\varsigma|^{2}-\langle v, \varsigma\rangle^{2}\right] \geq$

$$
\operatorname{Tr}\left(A(p) D^{2} \phi(p)\right)-C^{2} \frac{(|\nabla \phi(p)|+\omega)^{2}}{\phi(p)} .
$$

ProOF. Let $\left\{v_{1}, v_{2}, \ldots, v_{n}\right\}$ be an orthonormal basis in $\mathbb{R}^{n}$ of eigenvectors of $D^{2} u\left(P_{\tau}\right)$, with eigenvalues $\left\{\lambda_{1} \leq \lambda_{2} \leq \cdots \leq \lambda_{n}\right\}$. By Lemma A.1 with $G=$ $\left.D^{2} u\left(P_{\tau}\right)\right)$ we get

$$
\begin{aligned}
\operatorname{Tr}\left(B\left(P_{\tau}\right) D^{2}\left(P_{\tau}\right)\right) & \leq \beta_{n}\left(\sum_{i=1}^{n-1} \lambda_{i} \frac{\beta_{i}}{\beta_{n}}+\lambda_{n}\right) \\
& =\frac{\beta_{n}}{\left\langle F(p) v_{n}, v_{n}\right\rangle}\left(\sum_{i=1}^{n-1} \lambda_{i} \frac{\beta_{i}\left\langle F(p) v_{n}, v_{n}\right\rangle}{\beta_{n}}+\lambda_{n}\left\langle F v_{n}, v_{n}\right\rangle\right)
\end{aligned}
$$

Thus we ask that for $i=1,2, \ldots, n-1$

$$
\left\langle F(p) v_{i}, v_{i}\right\rangle=\frac{\beta_{i}\left\langle F v_{n}, v_{n}\right\rangle}{\beta_{n}}
$$

i.e.,

If $\langle v, \varsigma\rangle=0$, then

$$
\frac{\left\langle F(p) v_{i}, v_{i}\right\rangle}{\left\langle F(p) v_{n}, v_{n}\right\rangle}=\frac{\beta_{i}}{\beta_{n}}
$$

$$
F(p)=\left|A^{1 / 2}(p) \nabla \phi(p)\right|^{2} v \otimes v+\phi^{2}(p) \varsigma \otimes \varsigma+A(p)
$$


and

$$
\left\langle F(p) v_{i}, v_{i}\right\rangle=\sum_{k, l=1}^{n}\left(v_{k} v_{l}\left|A^{1 / 2}(p) \nabla \phi(p)\right|^{2}+\phi^{2}(p) \varsigma_{k} \varsigma_{l}+a_{k l}\right) v_{i k} v_{i l} .
$$

We can assume that $v=(0, \ldots, 0,1)$ and $\varsigma=\left(\varsigma_{1}, \varsigma_{2}, \ldots, \varsigma_{n-1}, 0\right)$. Thus for $i=1, \ldots, n$,

$$
\begin{aligned}
\left\langle F(p) v_{i}, v_{i}\right\rangle= & \sum_{k, l=1}^{n} \delta_{k l}\left|A^{1 / 2}(p) \nabla \phi(p)\right|^{2} v_{i k} v_{i l}+\sum_{k, l=1}^{n-1} \phi^{2}(p) \varsigma_{k} \varsigma_{l} \\
& +\sum_{k, l=1}^{n} a_{k l} v_{i k} v_{i l} \\
= & \left|A^{1 / 2}(p) \nabla \phi(p)\right|^{2} v_{i n}^{2}+\phi^{2}(p) \sum_{k, l=1}^{n-1} \varsigma_{k} \varsigma_{l} v_{i k} v_{i l} \\
& +\sum_{k, l=1}^{n} a_{k l} v_{i k} v_{i l} \\
= & \left|A^{1 / 2}(p) \nabla \phi(p)\right|^{2} v_{i n}^{2}+\phi^{2}(p) \sum_{k, l=1}^{n-1} \varsigma_{k} \varsigma_{l} v_{i k} v_{i l}+\left\langle A(p) v_{i}, v_{i}\right\rangle .
\end{aligned}
$$

From A.11 we get the following system, for $i=1,2, \ldots n-1$,

$$
\begin{aligned}
& \phi^{2}(p) \sum_{k, l=1}^{n-1}\left(v_{i k} v_{i l}-v_{n k} v_{n l} \frac{\beta_{i}}{\beta_{n}}\right) \varsigma_{k} \varsigma_{l}= \\
& \left|A^{1 / 2}(p) \nabla \phi(p)\right|^{2}\left(\frac{\beta_{i}}{\beta_{n}} v_{n n}^{2}-v_{i n}^{2}\right)+\left(\left\langle A(p) v_{n}, v_{n}\right\rangle \frac{\beta_{i}}{\beta_{n}}-\left\langle A(p) v_{i}, v_{i}\right\rangle\right),
\end{aligned}
$$

that is,

$$
\begin{aligned}
& \phi^{2}(p) \sum_{k, l=1}^{n-1}\left(v_{i k} v_{i l}-v_{n k} v_{n l} \frac{\beta_{i}}{\beta_{n}}\right) \varsigma_{k} \varsigma_{l}= \\
& \left|A^{1 / 2}(p) \nabla \phi(p)\right|^{2}\left(\frac{\beta_{i}}{\beta_{n}} v_{n n}^{2}-v_{i n}^{2}\right)+\left\langle A(p) v_{n}, v_{n}\right\rangle\left(\frac{\beta_{i}}{\beta_{n}}-\frac{\left\langle A(p) v_{i}, v_{i}\right\rangle}{\left\langle A(p) v_{n}, v_{n}\right\rangle}\right) .
\end{aligned}
$$

In particular, recalling that $\varsigma=\left(\varsigma_{1}, \varsigma_{2}, \ldots, \varsigma_{n-1}, 0\right)$, we obtain

$$
\phi^{2}(p)\left\langle\left[v_{i} \otimes v_{i}-\frac{\beta_{i}}{\beta_{n}} v_{n} \otimes v_{n}\right] \varsigma, \varsigma\right\rangle=
$$

$$
\left|A^{1 / 2}(p) \nabla \phi(p)\right|^{2}\left(\frac{\beta_{i}}{\beta_{n}} v_{n n}^{2}-v_{i n}^{2}\right)+\left\langle A(p) v_{n}, v_{n}\right\rangle\left(\frac{\beta_{i}}{\beta_{n}}-\frac{\left\langle A(p) v_{i}, v_{i}\right\rangle}{\left\langle A(p) v_{n}, v_{n}\right\rangle}\right) .
$$


From Lemma A.2 we get

$$
\left[\begin{array}{c}
v_{1}^{T} \\
\vdots \\
v_{n}^{T}
\end{array}\right]\left[v_{i} \otimes v_{i}-\frac{\beta_{i}}{\beta_{n}} v_{n} \otimes v_{n}\right]\left[v_{1}, \ldots, v_{n}\right]=e_{i} \otimes e_{i}-\frac{\beta_{i}}{\beta_{n}} e_{n} \otimes e_{n}
$$

As a consequence,

$$
\left[v_{i} \otimes v_{i}-\frac{\beta_{i}}{\beta_{n}} v_{n} \otimes v_{n}\right]=\left[v_{1}, \ldots, v_{n}\right]\left(e_{i} \otimes e_{i}-\frac{\beta_{i}}{\beta_{n}} e_{n} \otimes e_{n}\right)\left[\begin{array}{c}
v_{1}^{T} \\
\vdots \\
v_{n}^{T}
\end{array}\right] .
$$

Set

$$
w=\left[\begin{array}{c}
v_{1}^{T} \\
\vdots \\
v_{n}^{T}
\end{array}\right] \varsigma
$$

From (A.12), we deduce that for $i=1,2, \ldots, n-1$,

$$
\begin{aligned}
& \phi^{2}(p)\left\langle\left(e_{i} \otimes e_{i}-\frac{\beta_{i}}{\beta_{n}} e_{n} \otimes e_{n}\right) w, w\right\rangle= \\
& \left|A^{1 / 2}(p) \nabla \phi(p)\right|^{2}\left(\frac{\beta_{i}}{\beta_{n}} v_{n n}^{2}-v_{i n}^{2}\right)+\left\langle A(p) v_{n}, v_{n}\right\rangle\left(\frac{\beta_{i}}{\beta_{n}}-\frac{\left\langle A(p) v_{i}, v_{i}\right\rangle}{\left\langle A(p) v_{n}, v_{n}\right\rangle}\right) .
\end{aligned}
$$

Thus for $i=1,2, \ldots, n-1$

$$
\begin{aligned}
\phi^{2}(p)\left(w_{i}^{2}-\frac{\beta_{i}}{\beta_{n}} w_{n}^{2}\right)= & \left|A^{1 / 2}(p) \nabla \phi(p)\right|^{2}\left(\frac{\beta_{i}}{\beta_{n}} v_{n n}^{2}-v_{i n}^{2}\right) \\
& +\left\langle A(p) v_{n}, v_{n}\right\rangle\left(\frac{\beta_{i}}{\beta_{n}}-\frac{\left\langle A(p) v_{i}, v_{i}\right\rangle}{\left\langle A(p) v_{n}, v_{n}\right\rangle}\right) .
\end{aligned}
$$

In particular, for $i=1,2, \ldots, n-1$, we get

$$
\begin{aligned}
w_{i}^{2}=\frac{\beta_{i}}{\beta_{n}} w_{n}^{2}+\frac{1}{\phi(p)^{2}}[ & \left|A^{1 / 2}(p) \nabla \phi(p)\right|^{2}\left(\frac{\beta_{i}}{\beta_{n}} v_{n n}^{2}-v_{i n}^{2}\right) \\
& \left.+\left\langle A(p) v_{n}, v_{n}\right\rangle\left(\frac{\beta_{i}}{\beta_{n}}-\frac{\left\langle A(p) v_{i}, v_{i}\right\rangle}{\left\langle A(p) v_{n}, v_{n}\right\rangle}\right)\right] .
\end{aligned}
$$

Notice that selecting any $\varsigma=\left(\varsigma_{1}, \varsigma_{2}, \ldots, \ldots, \varsigma_{n-1}, 0\right)$ such that $\left\langle v_{n}, \varsigma\right\rangle=0$, then $w_{n}=0$. In this case we get

$$
\begin{aligned}
w_{i}^{2}=\frac{1}{\phi(p)^{2}}[ & \left|A^{1 / 2}(p) \nabla \phi(p)\right|^{2}\left(\frac{\beta_{i}}{\beta_{n}} v_{n n}^{2}-v_{i n}^{2}\right) \\
& \left.+\left\langle A(p) v_{n}, v_{n}\right\rangle\left(\frac{\beta_{i}}{\beta_{n}}-\frac{\left\langle A(p) v_{i}, v_{i}\right\rangle}{\left\langle A(p) v_{n}, v_{n}\right\rangle}\right)\right]
\end{aligned}
$$


As a consequence, we take, for $i=1,2, \ldots, n-1$,

$$
w_{i}=\frac{1}{\phi(p)} \sqrt{\left|\begin{array}{l}
\left|A^{1 / 2}(p) \nabla \phi(p)\right|^{2}\left(\frac{\beta_{i}}{\beta_{n}} v_{n n}^{2}-v_{i n}^{2}\right) \\
+\left\langle A(p) v_{n}, v_{n}\right\rangle\left(\frac{\beta_{i}}{\beta_{n}}-\frac{\left\langle A(p) v_{i}, v_{i}\right\rangle}{\left\langle A(p) v_{n}, v_{n}\right\rangle}\right)
\end{array}\right|}
$$

and $w_{n}=0$. Choosing

$$
\varsigma=\left[v_{1}, \ldots, v_{n}\right] w
$$

then A.8 follows.

We now estimate the quantity

$$
\delta(A, B)=\max _{i=1, \ldots, n-1}\left|\frac{\beta_{i}}{\beta_{n}}-\frac{\left\langle A(p) v_{i}, v_{i}\right\rangle}{\left\langle A(p) v_{n}, v_{n}\right\rangle}\right| .
$$

For $i=1, \ldots, n-1$ we write

$$
\begin{aligned}
\frac{\beta_{i}}{\beta_{n}}-\frac{\left\langle A v_{i}, v_{i}\right\rangle}{\left\langle A v_{n}, v_{n}\right\rangle}= & \left(\frac{\beta_{i}}{\beta_{n}}-\frac{\left\langle B v_{i}, v_{i}\right\rangle}{\left\langle B v_{n}, v_{n}\right\rangle}\right) \\
& +\frac{\left\langle B v_{i}, v_{i}\right\rangle\left\langle A v_{n}, v_{n}\right\rangle-\left\langle A v_{i}, v_{i}\right\rangle\left\langle B v_{n}, v_{n}\right\rangle}{\left\langle B v_{n}, v_{n}\right\rangle\left\langle A(p) v_{n}, v_{n}\right\rangle}
\end{aligned}
$$

where $B=B\left(P_{\tau}\right)$ and $A=A(p)$. Then

$$
\begin{aligned}
& \left|\frac{\beta_{i}}{\beta_{n}}-\frac{\left\langle v_{i}, v_{i}\right\rangle}{\left\langle A v_{n}, v_{n}\right\rangle}\right| \\
& \quad \leq\left|\frac{\lambda_{\max }(B)}{\lambda_{\min }(B)}-\frac{\lambda_{\min }(B)}{\lambda_{\max }(B)}\right|+\lambda_{\max }(A) \frac{|B-A|}{\lambda_{\min }(A) \lambda_{\min }(B)} \\
& \quad \leq \frac{1+\frac{\lambda_{\max }(B)}{\lambda_{\min }(B)}}{\lambda_{\min }(B)}\left(\lambda_{\max }(B)-\lambda_{\min }(B)\right)+\frac{\lambda_{\max }(A)}{\lambda_{\min }(A)} \frac{|B-A|}{\lambda_{\min }(B)} .
\end{aligned}
$$

Since

$$
\lambda_{\max }(B)-\lambda_{\min }(B) \leq 2|B-I|,
$$

keeping in mind A.14), we conclude that

$$
\begin{aligned}
& \left|\frac{\beta_{i}\left(P_{\tau}\right)}{\beta_{n}\left(P_{\tau}\right)}-\frac{\left\langle A(p) v_{i}, v_{i}\right\rangle}{\left\langle A(p) v_{n}, v_{n}\right\rangle}\right| \\
& \quad \leq 2 \frac{\lambda+\Lambda}{\lambda^{2}}|B-I|+\frac{\Lambda}{\lambda^{2}}|B-A| \\
& \quad \leq 2 \frac{\lambda+\Lambda}{\lambda^{2}}|B-I|+\frac{\Lambda}{\lambda^{2}}|B-I|+\frac{\Lambda}{\lambda^{2}}|A-I| \leq 6 \frac{\Lambda}{\lambda^{2}} \omega .
\end{aligned}
$$

Thus, with the previous choice of $\varsigma$, we infer

$$
|\varsigma|^{2} \leq|w|^{2} \leq C(\Lambda, n) \frac{|\nabla \phi(p)|^{2}+\delta(A, B)}{\phi(p)} \leq C \frac{|\nabla \phi(p)|^{2}+\delta(A, B)}{\phi(p)},
$$

which is A.9. 
Finally, from A.6 we obtain

$$
\begin{aligned}
\operatorname{Tr} & \left(A(p) D^{2} \phi(p)\right)-\phi(p)\left[|\varsigma|^{2}-\langle v, \varsigma\rangle^{2}\right] \\
& =\operatorname{Tr}\left(A(p) D^{2} \phi(p)\right)-\phi(p)|\varsigma|^{2} \\
& \geq \operatorname{Tr}\left(A(p) D^{2} \phi(p)\right)-C^{2} \phi(p)\left(\frac{|\nabla \phi(p)|+\delta(A, B)}{\phi(x)}\right)^{2} \\
& \geq \operatorname{Tr}\left(A(p) D^{2} \phi(p)\right)-C^{2} \frac{(|\nabla \phi(p)|+\omega)^{2}}{\phi(p)},
\end{aligned}
$$

that is A.10, concluding the proof.

Acknowledgments. F.F. and S.S. are partially supported by PRIN Project 2009 (MiUR) Italy, "Metodi di viscosità, geometrici e di controllo per modelli diffusivi nonlineari." F.F. was partially supported by the GNAMPA project "Equazioni non lineari su varietà: proprietà qualitative e classificazione delle soluzioni", by ERC Starting Grant Project 2011 EPSILON (Elliptic PDEs and Symmetry of Interfaces and Layers for Odd Nonlinearities), and by the University of Bologna, Italy.

\section{Bibliography}

[1] Argiolas, R.; Piro Grimaldi, A. Green's function, caloric measure and Fatou theorem for nondivergence parabolic equations in non cylindrical domains. Forum Math. 20 (2008), no. 2, 213 237. doi:10.1515/FORUM.2008.011

[2] Athanasopoulos, I. Free boundary regularity in Stefan type problems. Atti Accad. Naz. Lincei Cl. Sci. Fis. Mat. Natur. Rend. Lincei (9) Mat. Appl. 15 (2004), no. 3-4, 345-355.

[3] Athanasopoulos, I.; Caffarelli, L.; Salsa, S. Caloric functions in Lipschitz domains and the regularity of solutions to phase transition problems. Ann. of Math. (2) 143 (1996), no. 3, 413 434. doi: $10.2307 / 2118531$

[4] Athanasopoulos, I.; Caffarelli, L.; Salsa, S. Regularity of the free boundary in parabolic phasetransition problems. Acta Math. 176 (1996), no. 2, 245-282. doi:10.1007/BF02551583

[5] Athanasopoulos, I.; Caffarelli, L. A.; Salsa, S. Phase transition problems of parabolic type: flat free boundaries are smooth. Comm. Pure Appl. Math. 51 (1998), no. 1, 77-112. doi:10.1002/(SICI)1097-0312(199801)51:1<77::AID-CPA4>3.0.CO;2-C

[6] Caffarelli, L.; Salsa, S. A geometric approach to free boundary problems. Graduate Studies in Mathematics, 68. American Mathematical Society, Providence, R.I., 2005.

[7] Cerutti, M. C.; Ferrari, F.; Salsa, S. Two-phase problems for linear elliptic operators with variable coefficients: Lipschitz free boundaries are $C^{1, \gamma}$. Arch. Ration. Mech. Anal. 171 (2004), no. 3, 329-348. doi:10.1007/s00205-003-0290-5

[8] Fabes, E. B.; Garofalo, N.; Salsa, S. Comparison theorems for temperatures in noncylindrical domains. Atti Accad. Naz. Lincei Rend. Cl. Sci. Fis. Mat. Natur. (8) 77 (1984), no. 1-2, 1-12 (1985).

[9] Fabes, E. B.; Safonov, M. V. Behavior near the boundary of positive solutions of second order parabolic equations. Proceedings of the conference dedicated to Professor Miguel de Guzmán (El Escorial, 1996). J. Fourier Anal. Appl. 3 (1997), Special Issue, 871-882. doi:10.1007/BF02656492

[10] Ferrari, F.; Salsa, S. Regularity of the free boundary in two-phase problems for linear elliptic operators. Adv. Math. 214 (2007), no. 1, 288-322. doi:10.1016/j.aim.2007.02.004 
[11] Ferrari, F.; Salsa, S. Regularity of the solutions for parabolic two-phase free boundary problems. Comm. Partial Differential Equations 35 (2010), no. 6, 1095-1129. doi:10.1080/03605301003717126

[12] Friedman, A. The Stefan problem in several space variables. Trans. Amer. Math. Soc. 133 (1968), 51-87. doi:10.2307/1994932

[13] Garofalo, N. Second order parabolic equations in nonvariational forms: boundary Harnack principle and comparison theorems for nonnegative solutions. Ann. Mat. Pura Appl. (4) 138 (1984), 267-296. doi:10.1007/BF01762548

[14] Kim, I. C.; Požár, N. Viscosity solutions for the two-phase Stefan problem. Comm. Partial Differential Equations 36 (2011), no. 1, 42-66. doi:10.1080/03605302.2010.526980

[15] Koch, H. Classical solutions to phase transition problems are smooth. Comm. Partial Differential Equations 23 (1998), no. 3-4, 389-437. doi:10.1080/03605309808821351

[16] Krylov, N. V.; Safonov, M. V. A property of the solutions of parabolic equations with measurable coefficients. Izv. Akad. Nauk SSSR Ser. Mat. 44 (1980), no. 1, 161-175, 239.

[17] Ladyzhenskaya, O. A.; Ural'tseva, N. N. Linear and quasilinear elliptic equations. Academic Press, New York-London, 1968.

[18] Lu, G.; Wang, P. On the uniqueness of a solution of a two-phase free boundary problem. J. Funct. Anal. 258 (2010), no. 8, 2817-2833. doi:10.1016/j.jfa.2009.08.008

[19] Prüss, J.; Saal, J.; Simonett, G. Existence of analytic solutions for the classical Stefan problem. Math. Ann. 338 (2007), no. 3, 703-755. doi:10.1007/s00208-007-0094-2

[20] Salsa, S. Regularity in free boundary problems. Conf. Semin. Mat. Univ. Bari 270 (1997), 1-23.

[21] Widman, K.-O. Inequalities for the Green function and boundary continuity of the gradient of solutions of elliptic differential equations. Math. Scand. 21 (1967), 17-37 (1968).

FAUSTO FERRARI

Dipartimento di Matematica

Università di Bologna

Piazza di Porta S. Donato, 5

40126 Bologna

ITALY

E-mail: fausto.ferrari@

unibo.it

Received December 2011.

Revised November 2012.
SANDRO SALSA

Dipartimento di Matematica

Politecnico di Milano

Via Bonardi 7

20133 Milano

ITALY

E-mail: sandro.salsaepolimi.it 